\title{
Electrodynamics of an omega-band as deduced from optical and magnetometer data
}

\author{
H. Vanhamäki ${ }^{1}$, K. Kauristie ${ }^{1}$, O. Amm ${ }^{1}$, A. Senior ${ }^{2}$, D. Lummerzheim ${ }^{3}$, and S. Milan ${ }^{4}$ \\ ${ }^{1}$ Finnish Meteorological Institute, Arctic Research Unit, Helsinki, Finland \\ ${ }^{2}$ Department of Communication Systems, Lancaster University, Lancaster, UK \\ ${ }^{3}$ Geophysical Institute, University of Alaska, Fairbanks, AK, USA \\ ${ }^{4}$ Department of Physics and Astronomy, University of Leicester, Leicester, UK
}

Received: 30 April 2009 - Revised: 6 August 2009 - Accepted: 20 August 2009 - Published: 1 September 2009

\begin{abstract}
We investigate an omega-band event that took place above northern Scandinavia around 02:00-02:30 UT on 9 March 1999. In our analysis we use ground based magnetometer, optical and riometer measurements together with satellite based optical images. The optical and riometer data are used to estimate the ionospheric Hall and Pedersen conductances, while ionospheric equivalent currents are obtained from the magnetometer measurements. These data sets are used as input in a local KRM calculation, which gives the ionospheric potential electric field as output, thus giving us a complete picture of the ionospheric electrodynamic state during the omega-band event.

The overall structure of the electric field and field-aligned current (FAC) provided by the local KRM method are in good agreement with previous studies. Also the $\boldsymbol{E} \times \boldsymbol{B}$ drift velocity calculated from the local KRM solution is in good qualitative agreement with the plasma velocity measured by the Finnish CUTLASS radar, giving further support for the new local KRM method. The high-resolution conductance estimates allow us to discern the detailed structure of the omega-band current system. The highest Hall and Pedersen conductances, $\sim 50$ and $\sim 25 \mathrm{~S}$, respectively, are found at the edges of the bright auroral tongue. Inside the tongue, conductances are somewhat smaller, but still significantly higher than typical background values. The electric field shows a converging pattern around the tongues, and the field strength drops from $\sim 40 \mathrm{mV} / \mathrm{m}$ found at optically dark regions to $\sim 10 \mathrm{mV} / \mathrm{m}$ inside the areas of enhanced conductivity. Downward FAC flow in the dark regions, while upward currents flow inside the auroral tongue. Additionally, sharp conductance gradients at the edge of an auroral tongue are associated with narrow strips of intense FACs, so that a strip of down-
\end{abstract}

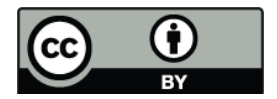

Correspondence to: $\mathrm{H}$. Vanhamäki (heikki.vanhamaki@fmi.fi) ward current flows at the eastern (leading) edge and a similar strip of upward current is present at the western (trailing) edge. The Joule heating follows the electric field pattern, so that it is diminished inside the bright auroral tongue.

Keywords. Ionosphere (Auroral ionosphere; Electric fields and currents)

\section{Introduction}

Omega-bands are meso-scale (usually $400-1000 \mathrm{~km}$ ) undulations in the poleward boundary of diffuse auroras. They typically appear in the morning sector of the auroral oval and during the recovery phase of auroral substorms. The eastward propagating auroral tongues interleaved with dark regions resemble the Greek letter $\Omega$, which has given the name for this quasi-periodic phenomenon.

The ionospheric electrodynamics of omega-bands has been studied in several papers (see e.g. Wild et al., 2000; Amm et al., 2005, and references therein), which all support the overall picture of a sequence of upward and downward field-aligned currents (FACs) located in the bright and dark regions of the structure, respectively. As the band structure with its relatively stationary current system moves above ground-based magnetometers, Ps6 type magnetic pulsations (period 4-40 min) are observed. The typical eastward drift velocity of omega-bands varies between $0.4-2 \mathrm{~km} / \mathrm{s}$.

The magnetospheric processes causing the field aligned current (FAC) system of omega-bands has also been addressed in several papers, but the general consensus about the primary source is still missing. Opgenoorth et al. (1994) associate omega-bands with energetic particle precipitation in the morning sector and suggest that both these phenomena have their origin at the outer edge of the ring current region. In the simulation of Yamamoto et al. (1997) diffuse auroras

Published by Copernicus Publications on behalf of the European Geosciences Union. 
are associated with a torus of hot plasma in the near-Earth magnetosphere and omega-bands are caused by the electrostatic interchange instability which can grow at the poleward (tailward) edge of the torus region during the substorm recovery phase. The 2-dimensional MHD-simulation runs by Janhunen and Huuskonen (1993) suggest that also the KelvinHelmholtz instability can cause some structures in the magnetic vorticity and FACs which resemble omega-bands.

In the omega-band cases analysed by Opgenoorth et al. (1983) and Amm (1996) the patterns of the converging (diverging) electric field within the auroral (dark) $\Omega$-regions are symmetric with respect to the vertical central axis of the tongue. Consequently, the superposition of omega-band and the morning sector electrojet appears as periodic undulations in the direction of horizontal total current. In a more recent case study presented by Amm et al. (2005) the effect is clearly different: In the presence of an omega-band the westward electrojet gets (partially) interrupted by alternating sheets of upward and downward FACs. The structure of electric field is a crucial factor to cause this behaviour. The field is not symmetric at the flanks of the auroral tongue, but has clearly smaller deviation in the East-West direction at the westward flank than in the eastward flank.

In the above described studies the magnetic and electric field observations have been used as the primary input data. Optical data have been used as reference information for mapping the deduced current and field structures against the visual structures. In the method of characteristics (e.g. Amm et al., 2005) optical data can also be used to estimate the Hall to Pedersen conductance ratio. In the present study we will revisit the omega-band electrodynamics once again with a method that uses magnetic, optical and riometer data as input for the calculation of electric fields and currents. Our procedure is a variant of the widely known KRM-method (Kamide et al., 1981), which has been modified by Vanhamäki and Amm (2007) for meso-scale analysis. We also utilize some semi-empirical models connecting observations of auroral luminosity and riometer absorption with height integrated conductance values (Senior et al., 2008).

The purpose of this paper is two-fold: Firstly, we want to present the first case study where the local KRM technique developed by Vanhamäki and Amm (2007) is applied to real data. This demonstrates the capabilities and limitations of the new analysis method, e.g. in respect to input data requirements. Secondly, we want to verify the reliability of the local KRM method against direct electric field measurements. Vanhamäki and Amm (2007) verified the new method using synthetic examples of typical ionospheric current systems, but in this study we compare the calculated electric field with direct measurements done by a coherent scatter radar.

In Sects. 2 and 3 we introduce our data analysis methods and the input data in more detail. Section 4 describes our event (9 March 1999, around 02:00 UT) and Sect. 5 shows the results from its analysis. The main findings of this study are discussed and summarised in Sect. 6.

\section{Data and analysis methods}

\subsection{Ionospheric equivalent currents}

We use magnetic data from the IMAGE magnetometer network, shown in Fig. 1. The ground magnetic measurements are converted into ionospheric equivalent currents with the SECS method, introduced by Amm and Viljanen (1999) and carefully tested by Pulkkinen et al. (2003). The background magnetic field is almost vertical in the area of the IMAGE network (inclination is $\sim 73^{\circ}$ in the southern parts), so we may assume that the equivalent currents are equal to the divergence-free part of the true ionospheric current (see e.g. Sect. 2.4.2 of Untiedt and Baumjohann, 1993).

Our analysis area is located at the northern mainland part of the IMAGE network, where the magnetometer network is densest. Consequently, we are able to calculate the equivalent current with spatial resolution of $0.4^{\circ}$ in latitude and $1.0^{\circ}$ in longitude. This corresponds to a $45 \times 40 \mathrm{~km}$ grid at latitude $69^{\circ} \mathrm{N}$. The temporal resolution of the magnetic data is $10 \mathrm{~s}$.

It should be noted that we have not separated the ground magnetic field into internal and external parts. During magnetically active periods significant part of the ground magnetic disturbance may be caused by internal induced currents (up to $40 \%$ during substorm onset, Tanskanen et al., 2001). However, the internal part of the magnetic field is generally much smoother than the external part. This means that our estimates of the ionospheric equivalent current may have slightly too large magnitude, but the spatial structure should be correct.

\section{2 "Energy map" conductance estimates}

Kosch et al. (2001) introduced a method to derive maps of the characteristic energy of precipitating electrons from cosmic noise absorption (CNA) and auroral optical intensity at $557.7 \mathrm{~nm}$. Senior et al. (2008) extended this "energy map" method so that also the flux of the precipitating electrons can be determined, assuming that the electrons have a Maxwellian energy spectrum. When both the characteristic energy and total energy flux of the precipitation are known, the ionospheric Hall and Pedersen conductances can be calculated by using the Robinson formulae (Robinson et al., 1987). These conductance estimates will be referred to as "emap" estimates.

In this study we use CNA measured by the IRIS imaging riometer (Browne at al., 1995) located at Kilpisjärvi (KIL in Fig. 1) and intensity-calibrated optical images from the Digital All-Sky Imager (DASI, Kosch et al., 1998) located at Skibotn (roughly between KIL and TRO in Fig. 1). The conductance estimates have high temporal (10 s) resolution in the area of $220 \times 220 \mathrm{~km}$ over the IRIS and DASI instruments. There are some time-steps when the emap estimate is not available for the whole analysis area. This is caused by the inherent uncertainty in estimating the quiet-day noise 


\section{IMAGE Magnetometer Network}

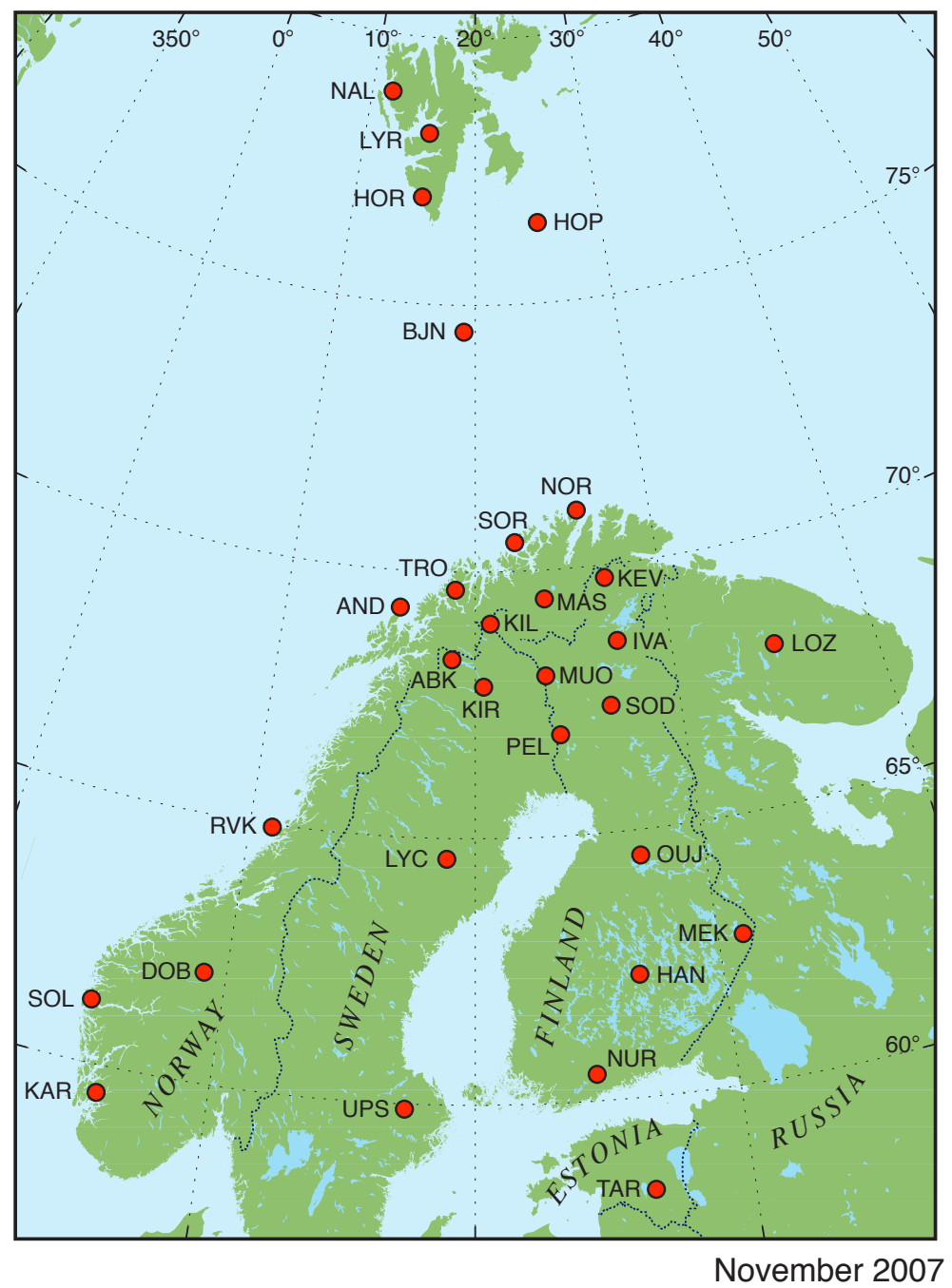

Fig. 1. The present extent of the IMAGE magnetometer network (see also http://space.fmi.fi/image/). During the omega-band event on 9 March 1999 data from TAR, DOB, RVK, LEK, IVA and MAS was not available.

level, leading to negative CNA values. These time-steps are omitted from the analysis. The emap estimate is given in a $10 \times 10 \mathrm{~km}$ grid, but the true spatial resolution of the estimate is variable, mainly due to the IRIS CNA measurements. IRIS has about $18 \mathrm{~km}$ resolution near the zenith over Kilpisjärvi, but the resolution decreases with increasing distance from the zenith. The DASI optical data are stored at $10 \mathrm{~km}$ resolution, and in the energy-map calculations the CNA measurements are interpolated onto the same $10 \mathrm{~km}$ grid.

Senior et al. (2008) compared the emap estimates and two other methods of determining conductances using riometer or optical data against EISCAT incoherent scatter radar measurements for two separate events. They found that for this particular event (9 March 1999) the DASI auroral $557.7 \mathrm{~nm}$ intensity values had to be scaled by a factor of 2.8 in order to match the EISCAT conductance measurement. The reason for this scaling is unclear, but possibly it was a calibration problem with the DASI instrument at this time. Senior et al. (2008) concluded that the emap method gave the best Hall conductance estimates (correlation 0.97-0.98 with EISCAT measurement), while the Pedersen conductance estimates obtained with the emap method were only slightly worse (correlation 0.88-0.90). During the studied intervals the EISCAT measurements were available mostly in the magnetic field aligned direction, but Senior et al. (2008) concluded that there was some indication that the evaluated methods (including the emap method) do not degrade noticeably for angles up to $\sim 20^{\circ}$ around the magnetic zenith, corresponding to a diameter of $\sim 40 \mathrm{~km}$ at $110 \mathrm{~km}$ altitude. 


\subsection{UVI conductance estimates}

The Ultra-Violet Imager (UVI) instrument onboard the POLAR spacecraft measures the auroral UV emission within the Lyman-Birge-Hopfield (LBH) band at 140-180 nm. Germany et al. (1997, 1998a,b) describe the method used to estimate the ionospheric conductances. Here we give just a brief outline.

The LBH band is divided into short and long wavelength parts, LBHS at $140-160 \mathrm{~nm}$ and LBHL at $160-180 \mathrm{~nm}$, respectively. At altitudes below $\sim 150 \mathrm{~km}$, emission in the LBHS band experiences larger absorption due to atmospheric $\mathrm{O}_{2}$. Thus the ratio between the LBHS and LBHL intensities depends on the altitude of the UV emission and can be used to estimate the characteristic energy of the precipitating electrons. The longer wavelength emission in the LBHL band is not significantly absorbed and is used to estimate the total precipitating energy flux in the energy range $0.1-20 \mathrm{keV}$. The ionospheric Hall and Pedersen conductances can be calculated by using the Robinson formulae (Robinson et al., 1987). These conductance estimates are will be referred to as "UVI" estimates.

The nominal spatial resolution of the UVI instrument is $\sim 40 \mathrm{~km}$ at apogee, but due to wobbling of the POLAR despun platform the resolution is reduced to $\sim 360 \mathrm{~km}$ in one direction. The temporal resolution of the UVI conductance estimate (the time required to take both LBHS and LBHL images) is $184 \mathrm{~s}$ for this specific event.

Aksnes et al. (2002) have shown that conductance estimates derived from only UVI data may underestimate the Hall conductance by several tens of percent. This is caused by the limited sensitivity to precipitating electrons with energies over $20 \mathrm{keV}$, which contribute to the Hall conductance. Most of the Pedersen conductance is caused by lower energy electrons and therefore the uncertainty in $\Sigma_{P}$ is significantly smaller.

\subsection{Electric field measurements}

Our analysis area is situated within the field of view of the STARE coherent scatter radar system (see e.g. Nielsen et al., 1999). STARE was still in operation during the event (it was decommissioned in 2005), but unfortunately there are no usable data for this period.

Also the CUTLASS coherent scatter radar system (see e.g. Lester et al., 2004) operates in this area. CUTLASS consists of two separate radars, one in Finland and the other in Iceland, that have an overlapping field-of-view. During the omega-band event only the Finnish radar received ionospheric backscatter from our analysis area. This means that we measure only the line-of-sight component of the plasma velocity, as seen from the Finnish radar.

During this event the CUTLASS radar operated in standard scan mode, meaning that the spatial resolution along each radar beam is $45 \mathrm{~km}$ and the whole field-of-view is scanned every $2 \mathrm{~min}$. Each radar beam is integrated for $7 \mathrm{~s}$, beginning from the westernmost beam. In Sect. 5.3 we compare the $\boldsymbol{E} \times \boldsymbol{B}$ drift velocity calculated from the KRM electric field (see Sect. 3.1) against the line-of-sight radar measurement.

\section{Calculating the ionospheric electric field}

\subsection{Local KRM method}

The ionospheric electric field can be calculated with the local KRM method introduced by Vanhamäki and Amm (2007), using the equivalent currents and conductance estimates as input data. The local KRM method uses the same input data as the traditional KRM method introduced by Kamide et al. (1981), but the mathematical formulation is quite different. The traditional KRM method can be used only in global (or hemispherical) studies, because in local scale studies the unknown boundary conditions at the edges of the analysis area may cause large uncertainty in the solution (Murison et al., 1985). The new method developed by Vanhamäki and Amm (2007) overcomes this difficulty by the different mathematical approach. In the meso-scale test cases considered by Vanhamäki and Amm (2007) the new local KRM method gave much more reliable results than the traditional KRM method.

It should be noted that in the local KRM method the ionospheric electric field is assumed to be a potential field, which means that inductive effects are neglected. Vanhamäki et al. (2007) showed that inductive effects may be locally important in some dynamical auroral systems. Their analysis included a data-based model of an omega-band (constructed by Amm, 1996), and the results indicated that in some relatively small $(<100 \mathrm{~km})$ "hot-spots" the induced rotational field may contribute up to $\sim 25 \%$ of the total electric field. However, when considered over the whole analysis area the inductive effect should be smaller than $1 \%$.

The local KRM formulation presented by Vanhamäki and Amm (2007) makes use of Cartesian geometry. This is a very reasonable approximation in the emap data area of $220 \times 220 \mathrm{~km}$. With the UVI conductance estimates we calculate the ionospheric electric field at area $66^{\circ}-72^{\circ} \mathrm{N}, 16^{\circ}-$ $30^{\circ} \mathrm{E}$, which correspond to about $700 \times 600 \mathrm{~km}$. Even in this larger area the curvature of the Earth may be neglected quite safely. It should be noted that in principle the local KRM method may also be formulated in spherical geometry, as indicated by Vanhamäki and Amm (2007), enabling study of larger scale current systems.

Vanhamäki and Amm (2007) tested the local KRM method using several realistic, data-based models of typical auroral current systems, including an electrojet, westward travelling surge and omega-band. Although the local KRM method performed better than the previous analysis methods, the errors in the solution were not too small. For example, in the omega-band case the local KRM method had $43 \%$ error 

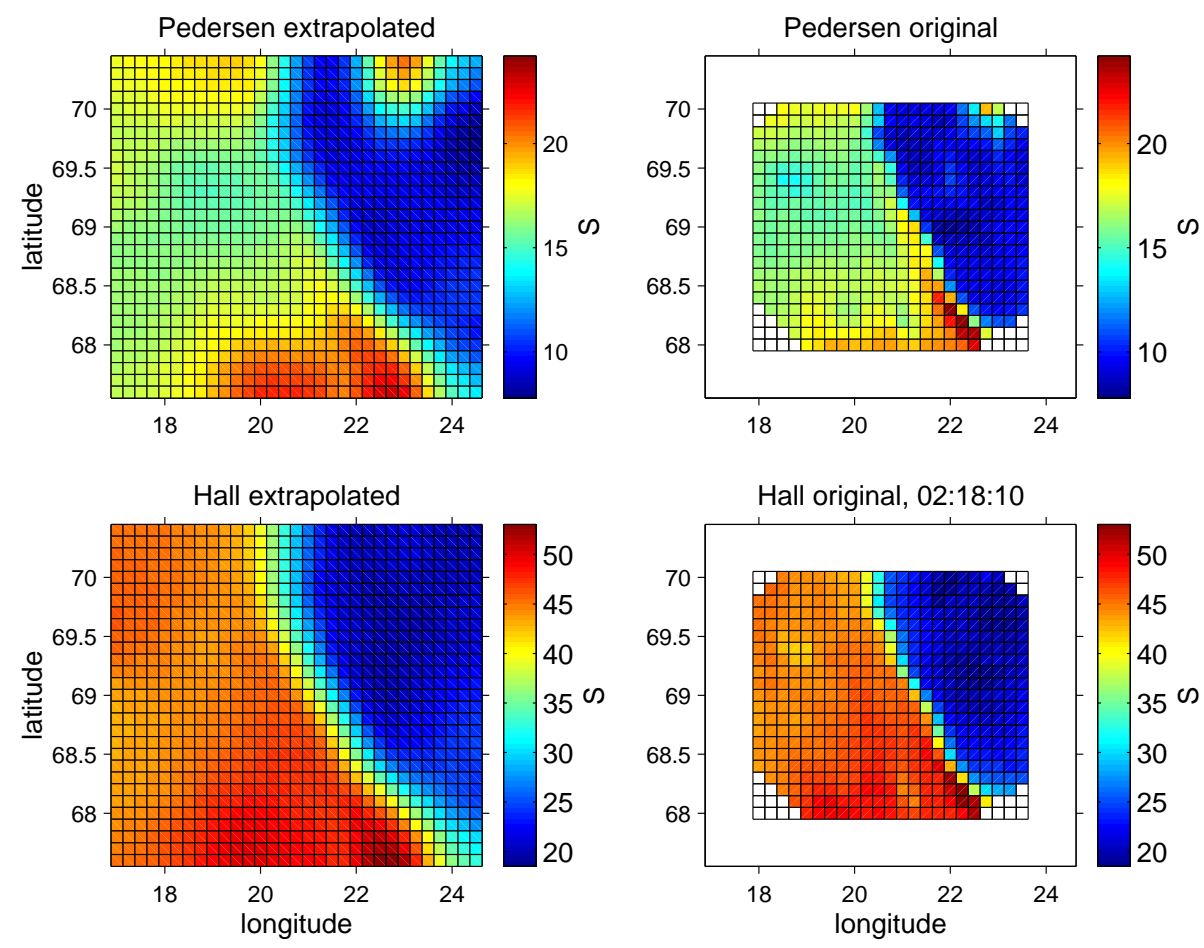

Fig. 2. Example of the original emap conductance data for one time step (panels on the right). In order to be used in the local KRM method the conductance data is extrapolated to a larger grid and smoothed to match the spatial resolution of the equivalent current data (panels on the left).

(using the error definition of Vanhamäki and Amm, 2007) in the electric field. However, Vanhamäki and Amm (2007) concluded that the overall structure of the solution, e.g. the general locations of upward and downward FAC, was quite reliable even if the exact numerical values were questionable. We recommend that the same precaution is used when interpreting the results shown in Sect. 5.

In the present study some additional caution should be used, when interpreting the KRM results obtained using the emap conductance estimates. The reason is that the emap conductance estimate has much better spatial resolution than the $\boldsymbol{J}_{e q}$ data (see Table 1), and consequently it contains more small scale structures. In the local KRM method this discrepancy, where small scale conductance gradients are not matched with changes in equivalent current, may result in spurious small scale structures in the FAC and, to a lesser extent, also in the electric field. This happens because the electric field is partially limited by the condition that it must be curl-free, but the FAC pattern, together with associated curlfree horizontal current, is the free parameter that is adjusted so that Ohm's law, ground magnetic observations and condition $\nabla \times \boldsymbol{E}=0$ are satisfied. We try to mitigate the situation by averaging the emap conductance estimate so that it matches the resolution of $\boldsymbol{J}_{e q}$ data more closely, as discussed in the next section. However, some differences remain and the FAC results displayed in Sect. 5 may contain some artificial features.

\subsection{Data processing}

We will apply the local KRM method to two data sets: 1) The large scale structure of the omega-band is obtained by using ground-based equivalent current $\boldsymbol{J}_{e q}$ and the UVI conductance estimate as input data, while 2) the small scale structure is obtained by combining $\boldsymbol{J}_{e q}$ with emap conductance estimate. These data sets have very different native spatial and temporal resolutions, as summarized in Table 1. It should be further noted that during this event the POLAR spacecraft was near apogee and Scandinavia was near the limb of the area visible to UVI, so the spatial resolution given in the table may be optimistic (see http://uvi.nsstc.nasa.gov/ for online images).

The equivalent current data and emap conductance estimates are calculated in geographical coordinate system, but the UVI conductance estimate is given in apex geomagnetic coordinates (Richmond, 1995). When we transform the UVI data to the geographical coordinate system we assume that the difference between apex coordinates and altitude adjusted corrected geomagnetic coordinates (AACGM, Baker and Wing, 1998) is negligible. Gasda and Richmond (1998) noticed that at auroral latitudes $\left(68^{\circ}\right.$ MLAT), the 
Table 1. The native spatial and temporal resolutions of the various data types.

\begin{tabular}{cccc}
\hline & $J_{e q}$ & UVI & emap \\
\hline Spatial resolution (lat $\times$ long) & $0.4^{\circ} \times 1.0^{\circ}$ & $0.5^{\circ} \times 3.75^{\circ}$ & $0.1^{\circ} \times 0.25^{\circ}$ \\
Temporal resolution & $10 \mathrm{~s}$ & $184 \mathrm{~s}$ & $10 \mathrm{~s}$ \\
\hline
\end{tabular}

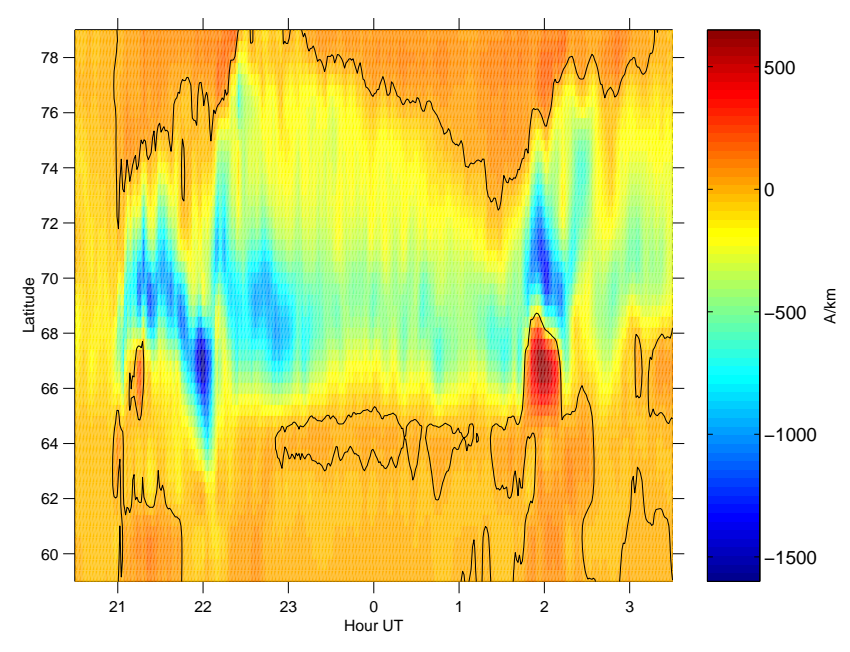

Fig. 3. The zonal component of the equivalent current across longitude $25^{\circ} \mathrm{E}$ as calculated from IMAGE magnetometers. Eastward current is positive and the black line is the zero-current contour.

maximum difference between the apex magnetic latitude at $110 \mathrm{~km}$ and corrected geomagnetic latitude at ground level is $0.17^{\circ}$, which is smaller than the UVI resolution. The UVI data are integrated for $184 \mathrm{~s}$, while the equivalent current data has time resolution of $10 \mathrm{~s}$. In the KRM calculation we simply took the instantaneous equivalent currents at the middle point of the UVI integration period, without averaging $\boldsymbol{J}_{e q}$ in time.

In order to make full use of the equivalent current data in the large scale KRM calculation, the UVI conductance estimate is interpolated to the denser $\boldsymbol{J}_{e q}$ grid (see Table 1). In the latitudinal direction the UVI resolution is already close to that of $\boldsymbol{J}_{e q}$ data, but in the longitudinal direction the UVI conductance estimate is heavily interpolated. This means that UVI data underestimate the amount spatial structure, especially in the longitudinal direction where the real UVI resolution is comparable to the size of individual $\Omega$-tongues. As a result, most of the electric field and FAC structure in the large scale KRM calculation, where UVI-based conductance data is used, stem from the equivalent current data. In this case the KRM algorithm does not generate artificial structure as happens in the opposite case where conductance data has more spatial structure than the equivalent current (see discussion of emap conductance data below).
Also the emap conductance estimate used in the more detailed small scale KRM calculation is processed, as illustrated in Fig. 2. The emap estimate is given in a $21 \times 23$ cell grid, with $\sim 10 \mathrm{~km}$ resolution. Due to the shape of the IRIS field-of-view the conductance estimate is not available in some grid cells at the corners of the area. The present version of the local KRM algorithm requires a regular rectangular grid, so we extrapolated the missing values using the nearest cells. Furthermore, in order to minimize possible boundary effects in the local KRM calculation, we extrapolated the conductance data 4 grid points $(\sim 40 \mathrm{~km})$ outwards in all directions. The local KRM calculations were performed in this larger $29 \times 31$ cell grid, but the results are shown only for the original area. Those time steps where any part of the emap conductance estimate is unavailable (see Sect. 2.2) were omitted from the analysis.

The emap conductance data has $\sim 10 \mathrm{~km}$ spatial resolution, compared with the $\sim 50 \mathrm{~km}$ resolution of the equivalent current data. In this case we found that simply interpolating $\boldsymbol{J}_{e q}$ to the denser grid produced unrealistic results in the local KRM method. Apparently the local KRM method does not work reliably when the conductance data has more small scale structure than the $\boldsymbol{J}_{e q}$ data, although in the opposite case (with UVI data) it seems to work quite well. Consequently, in addition to interpolating the $\boldsymbol{J}_{e q}$ data to the emap grid, we also smoothed the emap conductance data by taking running 5 -cell (about $50 \mathrm{~km}$ ) spatial averages. This way both data sets have roughly the same spatial resolution, and there is also a sufficiently large number of grid points in the calculation area. However, even after the averaging the emap conductance data has more small scale structure than the $\boldsymbol{J}_{e q}$ data, which results in spurious small scale structures in the FAC results displayed in Sect. 5. We try to identify these spurious features when discussing the results.

\section{Description of the event}

The early morning hours of 9 March 1999 were magnetically active since the recovery phase of a moderate substorm (AE $\sim 700 \mathrm{nT}$ ) was going on. The first onset of the substorm took place in the Fennoscandian sector around 21:00 UT (MLT $\approx \mathrm{UT}+2.5$ ) on $8 \mathrm{March}$. Inspection of the AE-index (data not shown here) reveals that in global scale the first onset of this substorm took place already around 19:30 UT. In the latitudeversus-time plot of equivalent currents (Fig. 3) the onset at 21:00 UT is visible as a sudden intensification and poleward 

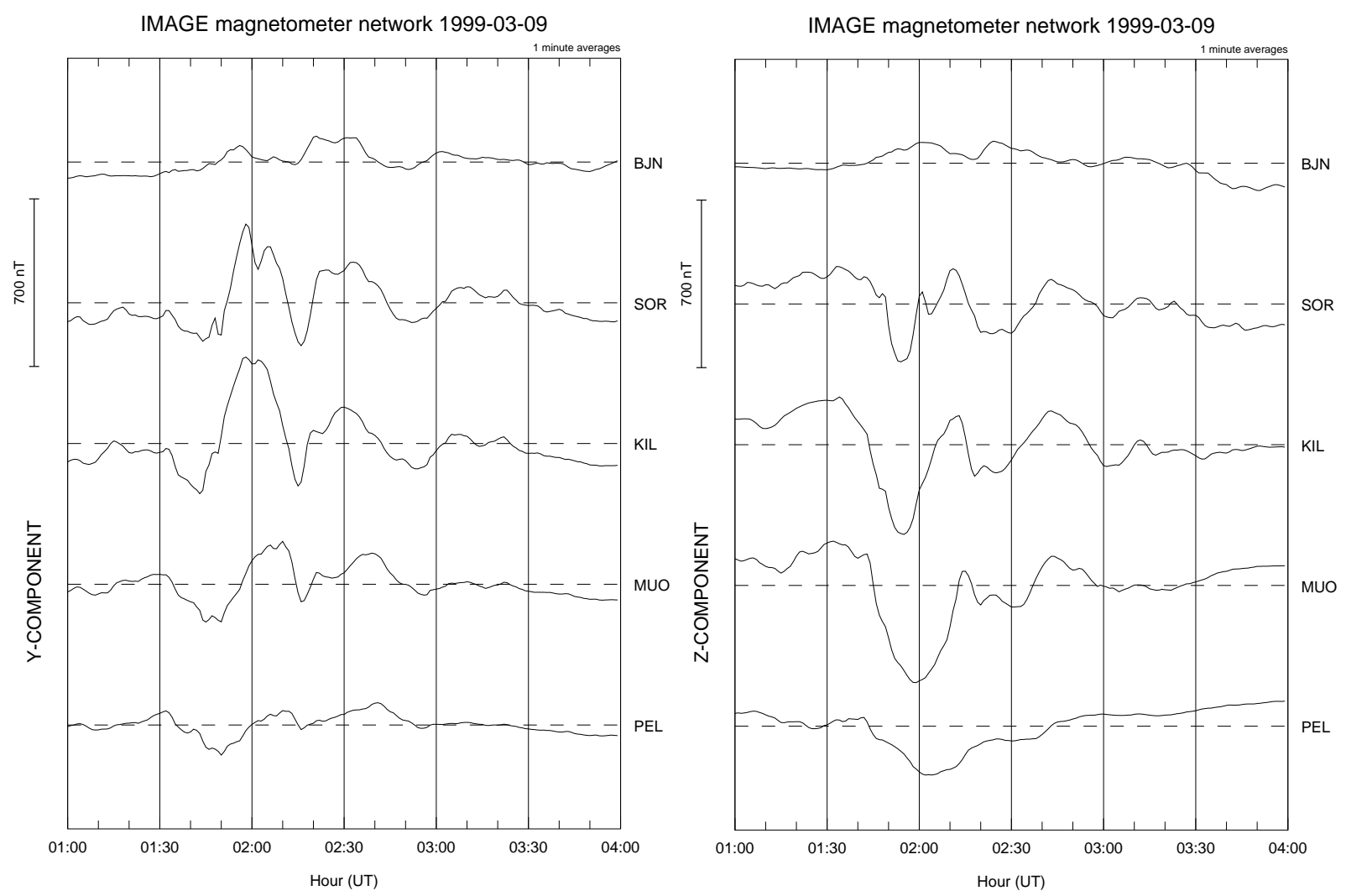

Fig. 4. Magnetogram showing the disturbance in the eastward (Y) and downward $(\mathrm{Z})$ components of the ground magnetic field at selected IMAGE stations.

motion of westward currents. After 21:30 UT the currents started to drift slowly equatorward which typically happens during growth phase but in this case the drift was only temporary and local as the next stronger onset took place at 22:00 UT. This second intensification propagated more polewards than the first one and reached also the IMAGE stations in Svalbard (magnetic latitude $\sim 75^{\circ}$ ). After 23:00 UT the recovery phase started, and consequently the westward electrojet weakened and narrowed. The electrojet activity woke up again at the arrival of the omega-band around 01:45 UT and the current fluctuations lasted until 03:00 UT. The IMAGE magnetograms in Fig. 4 show Pc6-type activity during this period, with at least two wave periods clearly discernible in all three magnetic field components. The amplitude of the pulsations $(\leq 400 \mathrm{nT})$ is larger in the $\mathrm{Z}$ and $\mathrm{Y}$ components than in the $\mathrm{X}$ component ( $\mathrm{X}$ not shown).

All-Sky Camera observations from station KIL (see Fig. 1) reveal that the auroral tongues in our omega-band event were not similar with each other. The sky was partly cloudy above KIL until 01:40 UT but after that the visibility improved so much that the first $\Omega$-tongue is clearly visible in the KIL ASC-images taken during 01:45-02:10 UT (data not shown here). The first auroral tongue was very large and had rich internal structure. In fact the electrojet behaviour (see Fig. 3) during the passage of the tongue contains some features typical for substorm onset intensifications: strong poleward expansion of the electrojet region and eastward equivalent currents in the southern stations (OUJ, HAN, NUR) simultaneously with the westward electrojet intensification. However, the UVI images do not show a global expansion phase during this time, so we interpret it as a pseudo-breakup that occurred simultaneously with the omega-band event.

It is interesting to note that after the pseudo-breakup the omega-band activity continued as if nothing special had happened. The second large auroral tongue drifted above KIL during 02:15-02:35 UT. This is covered in the ASC images shown in Fig. 5 and is more carefully analyzed in Sect. 5. The drift speed inferred from the ASC images was around $1.2 \mathrm{~km} / \mathrm{s}$, in the typical range for omega-bands. After 02:40 UT some eastward drifting structures (hooks and North-South directed arcs) were still observed but their resemblance with the letter $\Omega$ was not that evident any more. 

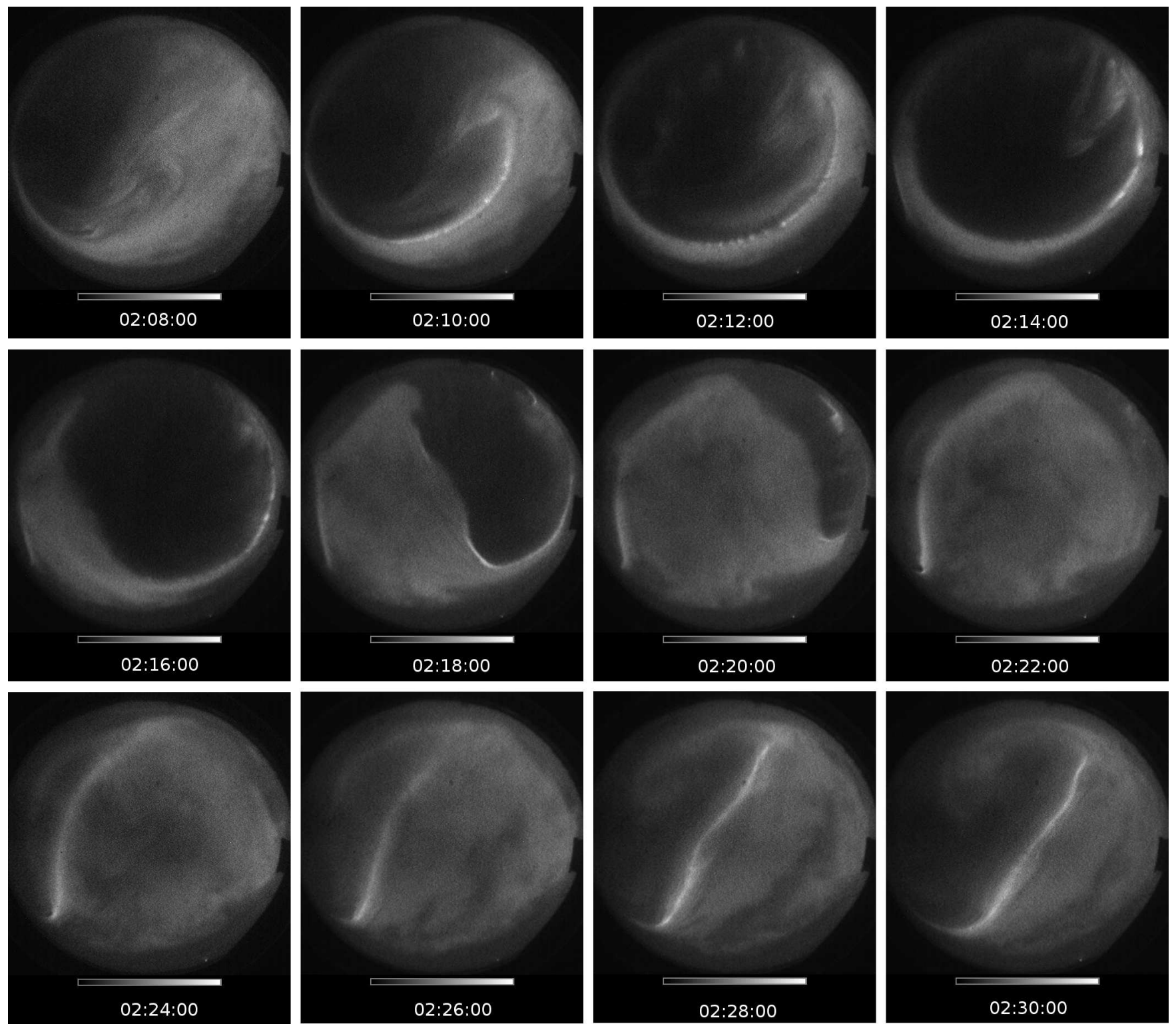

Fig. 5. Series of all-sky camera images taken at Kilpisjärvi showing the propagation of the omega-band directly over the station. Images are shown for every 2 min during the intervall 02:08-02:30 UT. Image orientation is such that North is up and East is right.

\section{Electrodynamics of an omega-band}

\subsection{Large scale structure with UVI conductance estimate}

Figure 6 shows a snapshot of the ionospheric electrodynamic variables at 02:17:15 UT (middle point of the $184 \mathrm{~s}$ integration period of the UVI data), corresponding to the passage of the second auroral tongue. The equivalent currents (top left panel, derived from ground magnetic data) together with Hall and Pedersen conductances (top and middle right, respectively, derived from UVI images) are used as input in the local KRM method. The ionospheric electric field (bottom left) as well as horizontal and field-aligned currents (bottom right; white arrows show the horizontal current while FAC is color coded) are obtained from the KRM calculation.

The red rectangle in the upper left panel of Fig. 6 is centered at Kilpisjärvi and shows the area where the more detailed emap conductance estimate is available (see the next section for results based on the emap estimate). According to the ASC images in Fig. 5 (1st and 2nd images in the middle row), the eastern edge of the bright tongue of the omegaband is near Kilpisjärvi zenith at 02:17:15 UT. This is in good agreement with the equivalent current, electric field and FAC distributions shown in Fig. 6. 

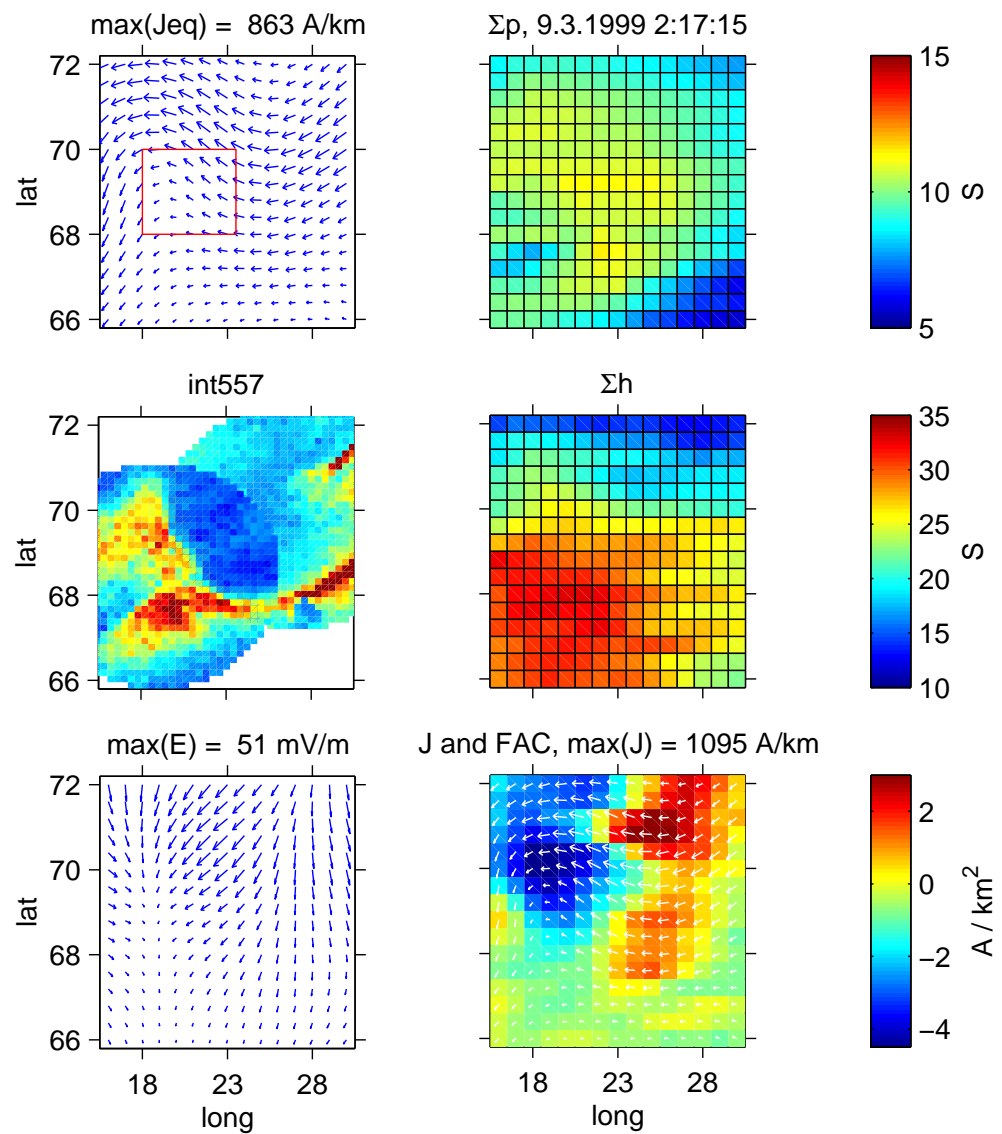

Fig. 6. Snapshot of ionospheric electrodynamic variables at 02:17:15 UT. Input data used in the local KRM method: Equivalent current Jeq, Pedersen and Hall conductances. Output of the KRM method: Ionospheric electric field E, horizontal current J and field-aligned current FAC. Positive FAC is downwards. Additionally, also the auroral intensity at $557 \mathrm{~nm}$ is shown in the middle left panel (cameras at ABK and KEV stations in Fig. 1, intensity is uncalibrated so no scale is shown). Here we use the UVI conductance estimate. The smaller area where the emap conductance estimate is available is indicated by the red rectangle in the top left panel.

The electric field obtained from the large scale KRM calculation shows a clear converging pattern on the eastward, northward and westward sides of the bright auroral tongue, which is typical for the omega-band (e.g. Untiedt and Baumjohann, 1993). The magnitude of $\boldsymbol{E}$ is around 40$50 \mathrm{mV} / \mathrm{m}$ in the dark regions of the omega-band, but drops down to $10-20 \mathrm{mV} / \mathrm{m}$ within the tongue. The converging electric field pattern and regions of upward and downward FAC regions line up with the auroral intensity data from ground based ASC. The Hall and Pedersen conductance estimates derived from UVI data do show enhancement by a factor $\sim 2$ in the general area of the omega-band. However, one would expect to see enhanced conductances in the upward FAC region and clearly diminished conductances in areas of strong downward FAC, but this is not the case in Fig. 6 . Moreover, the areas of enhanced Hall and Pedersen conductances are not completely overlapping, especially in the region $70^{\circ}-72^{\circ} \mathrm{N}$. These inconsistencies may be explained by the limited spatial resolution of the UVI instrument (espe- cially in the longitudinal direction, see Table 1) and by the fact that the analysis area was near the limb of UVI image. However, the general structure of the omega-band seems to appear quite well in these results, indicating that in this case most of the electric field and FAC structure seen in the KRM results stems from the equivalent current data.

The current system of the omega-band is superposed on top of a westward electrojet, and the equivalent current shows sinusoidal undulations around the bright and dark areas of the omega-band. The FAC shows alternating regions of upward and downward currents, so that upward current is located at the bright tongue of the omega-band and downward currents flow in the dark area between the tongues. As a result, the true horizontal current density (shown as white arrows in the bottom right panel of Fig. 6) does not undulate as strongly as the equivalent current, but rather the westward current intensifies and diminishes alternatingly. However, in this event the interruption of the westward electrojet is not as strong as in the event studied by Amm et al. (2005). 

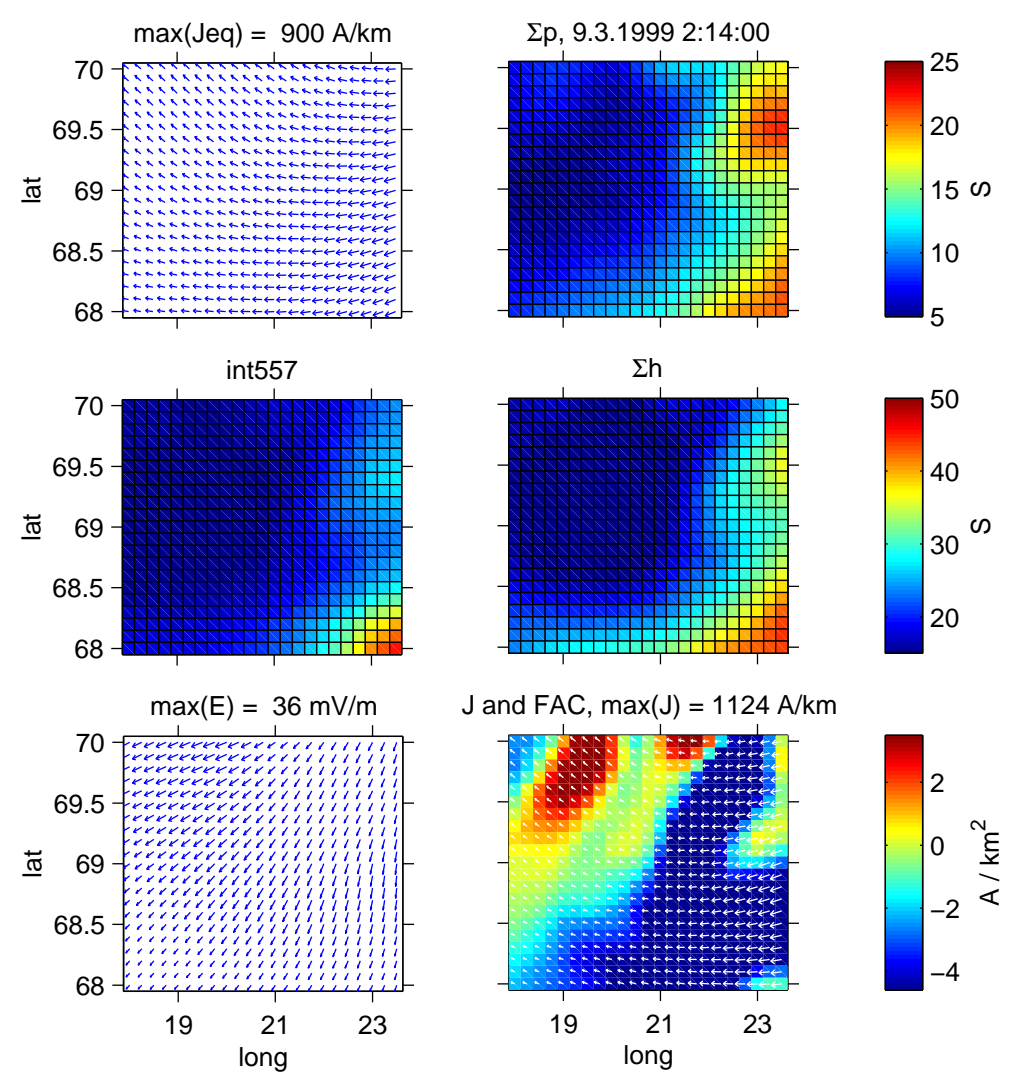

Fig. 7. Similar to Fig. 6, but here we use the emap conductance estimate and time is 02:14:00 UT. Here the auroral $557 \mathrm{~nm}$ intensity is from Kilpisjävi station, corresponding to Fig. 5. The area shown in this figure is indicated by the red rectangle in the top left panel of Fig. 6 .

\subsection{Small scale structure with emap conductance estimate}

Figures 7-10 show the more detailed view of omega-band electrodynamics obtained using the emap conductance estimate in the local KRM method. These snapshots cover the passage of the second bright auroral tongue over the emap observational area (see Fig. 6 for comparison with UVI data). In Fig. 7 at 02:14 UT the analysis area is situated almost completely in the dark region between the first and second tongues. In the next figure, Fig. 8 at 02:18 UT the second tongue covers the western part of the area and in Fig. 9 at 02:22 UT the tongue has moved eastward to cover the whole analysis area. Finally in Fig. 10 the trailing edge of the tongue enters the analysis region, so that the North-West corner of the area is already in the dark region.

The more detailed view given in Figs. 7-10 supports the overall picture of omega-band electrodynamics presented in previous studies and also in Fig. 6 using the larger scale UVI data set. The Hall and Pedersen conductances are enhanced in the bright auroral tongue, while the electric field shows a converging pattern around the tongue. Largest downward FAC flow in the dark regions (see Figs. 7 and 10) and upward FACS flow inside the auroral tongue (Fig. 9).
As discussed in Sect. 3, the emap conductance estimate has better spatial resolution than the equivalent current data, which may lead to spurious small scale structures in the output of the local KRM method, especially in the FAC distribution. For example, the rather small regions of intense downward FAC near the North-West corners of the analysis area in Figs. 7 and 10 are somewhat suspicious. These "blobs" of downward FAC are situated in the dark low-conductance areas, which is natural enough, but in both cases the downward FAC is associated with rather local conductance gradients that are not connected to the larger scale structure. Additionally, distinct features near the edges of the analysis area, such as the small patch of downward FAC in the North-East corner of Fig. 8, should be interpreted with caution, as they may be spurious features produced by boundary effects or extrapolation of the conductance estimates.

Despite the above cautions, there are some things we can say about the internal structure of the omega-band. The conductance gradient around the auroral tongue seem to be very sharp, as is evident in Figs. 8 and 10. It also appears that the largest Hall and Pedersen conductances occur at the edges of the auroral tongue. The Pedersen conductance shows a local minimum inside the tongue (see Fig. 9) and enhancement at 

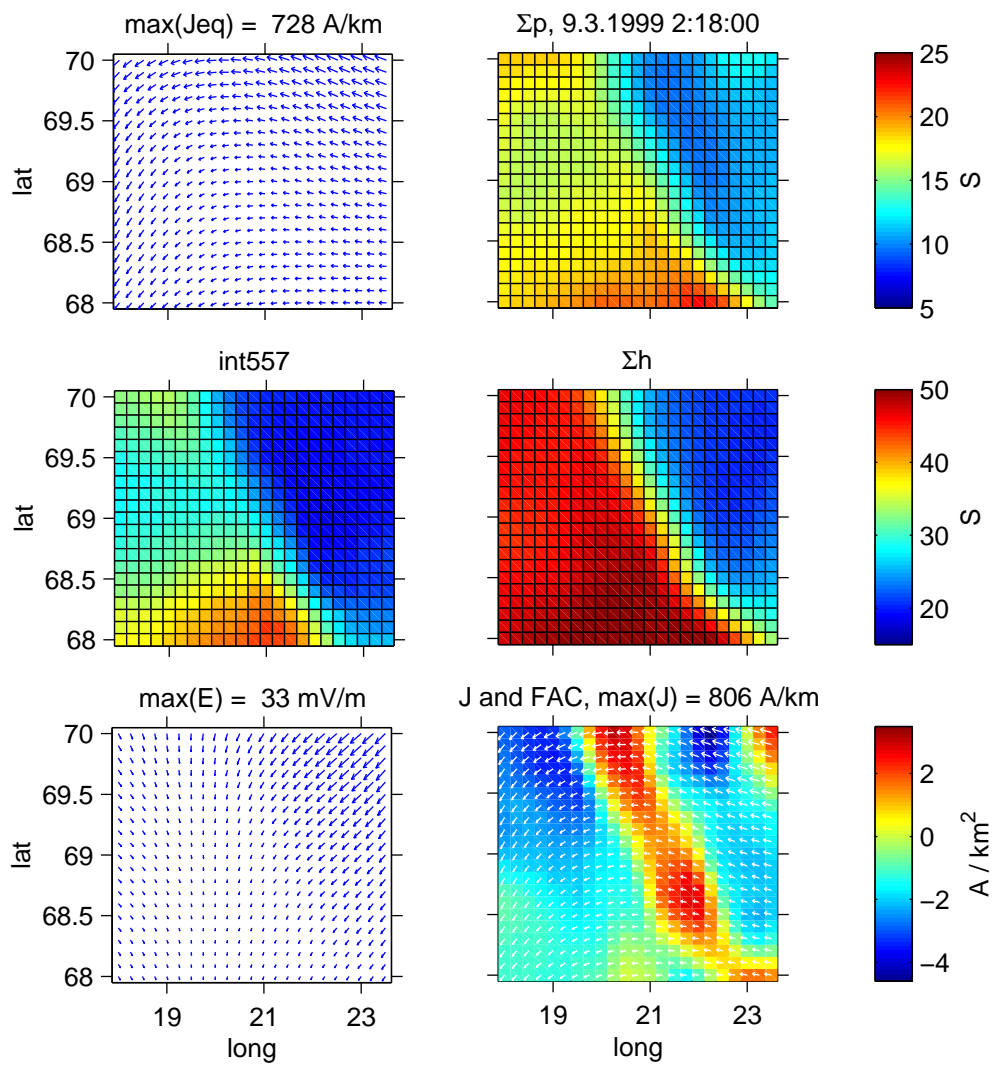

Fig. 8. Similar to Fig. 7, but for 02:18:00 UT.

the leading and trailing edges (Figs. 8 and 10, respectively). This is in harmony with the ASC images shown in Fig. 5, where the $\Omega$-shaped auroral tongue is slightly darker in the central region than at the edges (especially the trailing edge is clearly defined), as well as with previous observations (e.g. Wild et al., 2000; Opgenoorth et al., 1994).

The sharp conductance gradients are associated with strong FAC, so that a strip of downward FAC flows at the leading edge of the auroral tongue, while a similar strip of upward FAC is situated at the trailing edge. These conductance gradients are part of a larger structure and there are associated features in the equivalent current data, so we may be quite confident that they are real and not results of mismatched spatial resolution in the input data. Inside the auroral tongue (Fig. 9) FAC is dominantly upward, as expected, although weak downward FAC are present at the western parts of the tongue. The different ionospheric sources of FAC are discussed in more detail in the next section.

\subsubsection{Components of FAC}

Field-aligned currents are equal to the divergence of the horizontal sheet current, $\mathrm{FAC}=\nabla \cdot \boldsymbol{J}$. Using ionospheric Ohm's law we can further decompose FAC into sum of three terms
$\mathrm{FAC}=\Sigma_{P} \nabla \cdot \boldsymbol{E}+\nabla \Sigma_{P} \cdot \boldsymbol{E}-\left(\nabla \Sigma_{H} \times \boldsymbol{E}\right)_{z}$.

Here we have assumed that the electric field is curl-free, as is the case in the local KRM results.

Figure 11 shows an example of the total FAC and of the three different components at 02:18 UT. In this case there is a clear division so that the upward (negative) FAC stems from the divergence of the electric field, whereas the gradients of Hall and Pedersen conductance form the strip of downward FAC. Comparison with the ASC image shown in Figs. 5 and 8 reveals that the North-South aligned strip of downward FAC is located at the eastern (leading) edge of the auroral tongue. For other regions of the omega-band the FAC division is not shown, but inside the auroral tongue most of the FAC is associated with $\nabla \cdot \boldsymbol{E}$, as the conductance gradients are rather weak there. At the trailing edge the conductance gradients are strong, but the upward FAC driven by $\nabla \Sigma_{H}$ almost cancels the downward FAC associated with $\nabla \Sigma_{P}$, so the narrow strip of upward FAC visible in Fig. 10 is driven mainly by $\nabla \cdot \boldsymbol{E}$.

Figure 12 shows time series of the total upward and downward FAC integrated over the emap data area, as well as the division of FAC into different components. The area where the emap conductance estimate is available is about $220 \times 220 \mathrm{~km}$, which is smaller than the spatial extent of the 

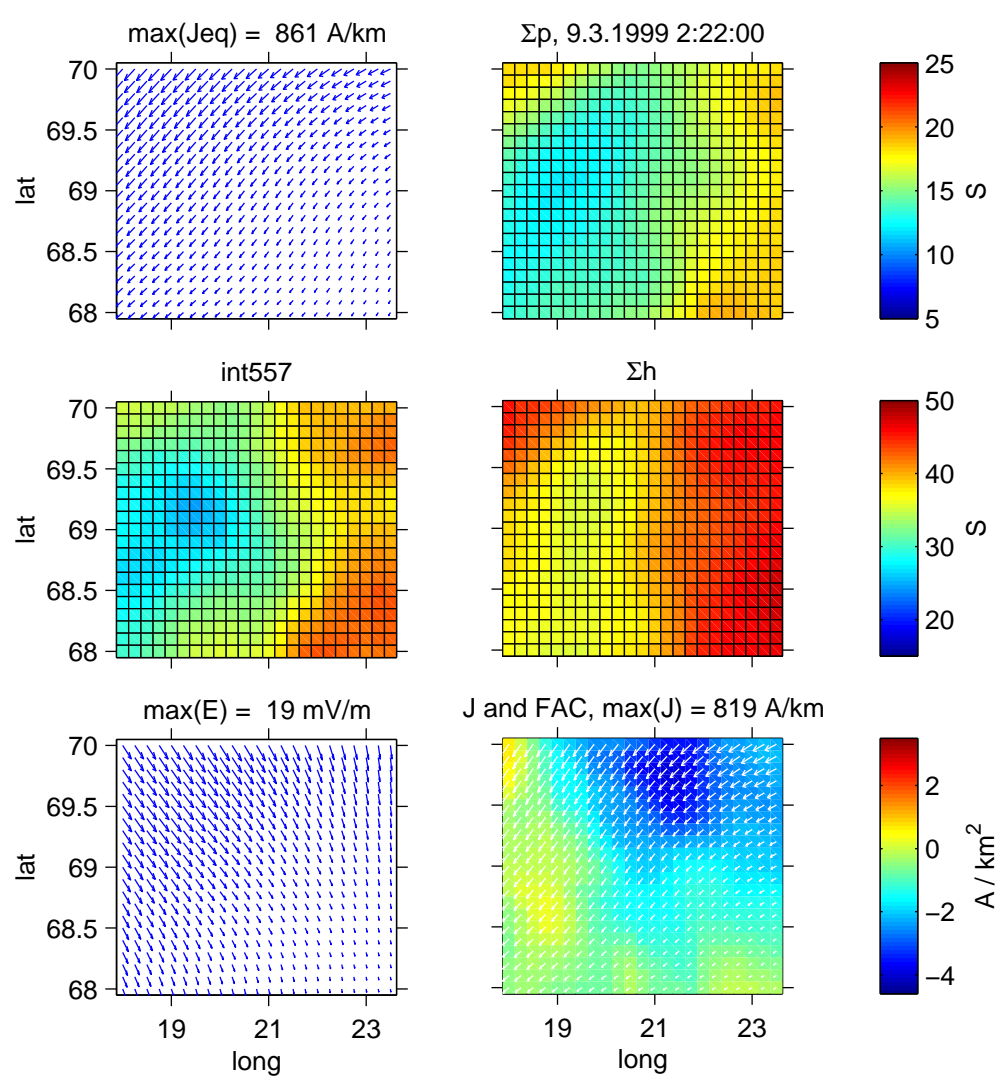

Fig. 9. Similar to Fig. 7, but for 02:22:00 UT.

omega-band. The omega-band is fairly stationary in the moving reference frame, as can be seen in Fig. 5. Based on visual inspection of the time series of KRM results (as in Figs. 710 , with 10 s resolution), we conclude that a major part of the variations in Fig. 12 reflect spatial differences between various parts of the omega-band, as they move over the area. This is shown in Fig. 12 by dividing the time series to five parts, labelled A-E. The transition from one region to another is gradual, so the times given in the figure are only indicative. However, there are also some real temporal variations in the FAC pattern, most importantly around 02:18 UT at the leading edge of the auroral tongue.

According to Fig. 12 there is a net upward FAC in the emap data area during the whole omega-band event. The downward FAC increases around 02:09-02:12 UT, when the previous auroral tongue is giving way to the dark region of the omega-band. However, at the same time also the upward current increases, so that the net current does not change markedly. Around 02:16 the second bright auroral tongue begins to cover the area, and the upward FAC decreases. At the same time the downward current intensifies, so that in the interval 02:18-02:21 UT the upward and downward currents are nearly in balance. The decrease of upward FAC is mostly due to the movement of the omega-band, but the strip of downward FAC at the leading edge of the tongue (see Fig. 8) intensifies markedly while moving eastward together with the omega-band. The intensification is caused mainly by the changing direction of the equivalent current with respect to the conductance gradient along the edge of the auroral tongue. The abrupt decrease of downward FAC at 02:20 UT is caused by the leading edge of the tongue leaving the analysis area. After this the downward current is very small and also the upward current stays almost constant as the auroral tongue and the trailing edge move over the analysis area.

The middle and bottom panels of Fig. 12 show the relative contributions of the three components in Eq. (1) to the downward and upward FAC, respectively. The upward current shows a quite simple pattern, where the gradient of Hall conductance dominates in the beginning of the omega-band event (i.e. at the trailing edge of the 1 st tongue and in the dark region between the tongues) while divergence of the electric field is most important term during the latter half of the event (i.e. at the leading edge and within the tongue). The contribution from the gradient of Pedersen conductance remains below 25\% for the whole period 02:06-02:30 UT. The transition from $\nabla \Sigma_{H}$ dominated upward FAC to $\nabla \cdot \boldsymbol{E}$ dominated occurs around 02:16 UT, when the leading edge of the second auroral tongue begins to enter the analysis region. 

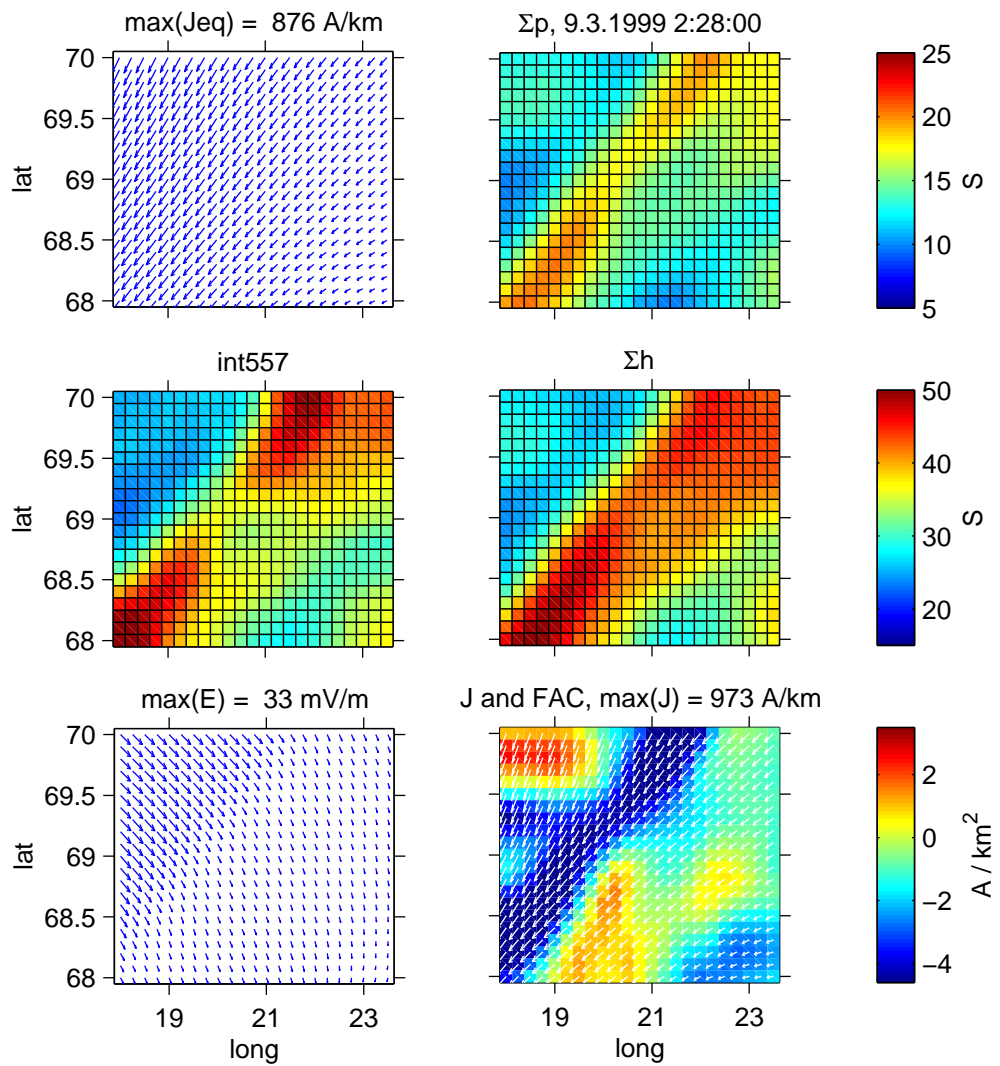

Fig. 10. Similar to Fig. 7, but for 02:28:00 UT.

The composition of downward FAC seems to be more complicated. During the interval 02:06-02:10 UT, i.e. when the trailing edge of the first auroral tongue is leaving the analysis area, $\nabla \Sigma_{P}$ is the most important contributor of downward FAC. Around 02:10 UT the contribution from $\nabla \Sigma_{P}$ starts to decrease and $\nabla \cdot \boldsymbol{E}$ becomes the dominant term for some minutes, when the analysis area is covered by the dark area of the omega-band. As the leading edge of the second auroral tongue starts to move across the analysis area, the contribution from $\nabla \Sigma_{H}$ increases and reaches $\sim 75 \%$ around 02:20 UT, when the whole area is inside the tongue. At the same time the role of $\nabla \cdot \boldsymbol{E}$ as the source of downward FAC decreases to almost zero. After about 02:20 UT there are large variations in the relative contribution from $\nabla \Sigma_{H}$ and $\nabla \Sigma_{P}$, but the total amount of downward FAC is so small that these variations probably are not meaningful.

\subsubsection{Joule heating}

Ionospheric Joule heating $P_{\text {Joule }}=\boldsymbol{E} \cdot \boldsymbol{J}$ is an important aspect of the energy flow in the coupled magnetosphere-ionosphere system. Figure 13 shows a snapshot of the ionospheric electric field, Pedersen conductance and Joule heating at 02:18 UT, while Fig. 14 shows a time series of the Joule heating integrated over the emap data area as well as the corre- lation coefficients between Joule heating and Pedersen conductance or electric field. The correlation coefficients are calculated over the emap analysis area, separately for each time step. Also here the time series reflects the properties of the various parts of the omega-band, as they move over the analysis area, rather than indicating temporal evolution of the omega-band as a whole.

The example shown in Fig. 13 demonstrates a strong correlation between the Joule heating and electric field intensity, whereas Joule heating and Pedersen conductance seem to anticorrelate. The time series displayed in the upper panel of Fig. 14 shows that the integrated Joule heating in the emap analysis area has peak value of $\sim 800 \mathrm{MW}$ at 02:06 UT, when the trailing edge of the first auroral tongue was about to leave the area. During the passage of the second tongue the integrated Joule heating decreased steadily, and reached minimum of $\sim 100 \mathrm{MW}$ around 02:20, when the analysis area was covered by the bright auroral tongue. Afterwards Joule heating increased slowly, as the second tongue moved again out of the area.

The lower panel of Fig. 14 shows that the correlation between Joule heating and electric field or Pedersen conductance changed markedly during the event. During interval 02:06-02:14 UT, as the first auroral tongue gives 

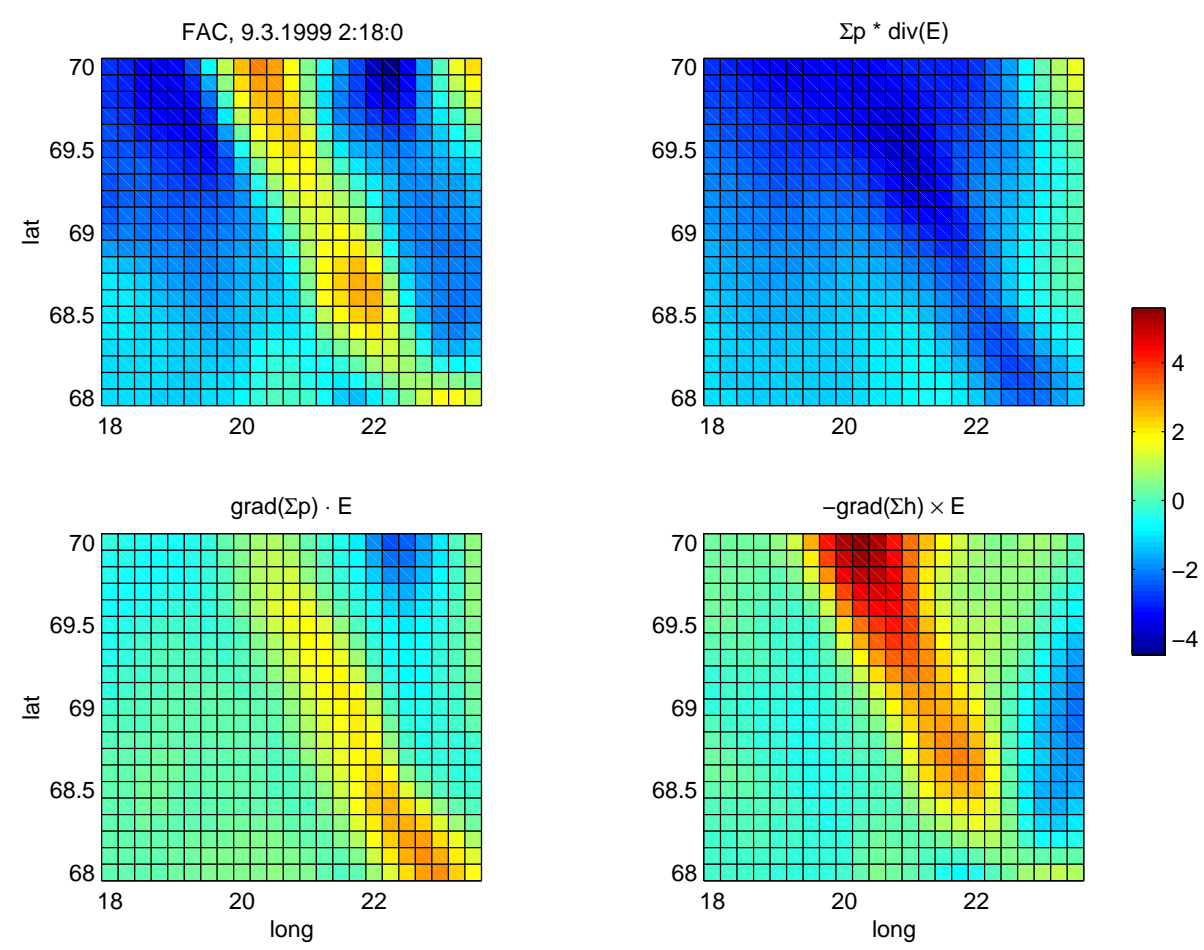

Fig. 11. Example of the total FAC and contributions from different terms. This snapshot corresponds to the situation illustrated in Fig. 8 .

way to the dark area of the omega-band, the correlation $\operatorname{corr}\left(P_{\text {Joule }},|\boldsymbol{E}|\right)$ decreases to 0.15 while $\operatorname{corr}\left(P_{\text {Joule }}, \Sigma_{P}\right)$ increases to 0.75 . Around 02:14 there is a sharp transition, so that $\operatorname{corr}\left(P_{\mathrm{J} o u l e}, \Sigma_{P}\right)$ changes sign from strong correlation to moderate anticorrelation and $\operatorname{corr}\left(P_{\text {Joule }},|\boldsymbol{E}|\right)$ increases to 0.95 in couple of minutes. This transition occurs when the analysis area is still covered by the dark region, but the leading edge of the second auroral tongue is about to enter the analysis area. During the rest of the event $\operatorname{corr}\left(P_{\text {Joule }},|\boldsymbol{E}|\right)$ stays high, around 0.95 . The correlation $\operatorname{corr}\left(P_{\mathrm{Joule}}, \Sigma_{P}\right)$ remains around -0.5 during the time when the leading edge of the tongue moves over the anaysis area. Inside the auroral tongue $\operatorname{corr}\left(P_{\mathrm{J} o u l e}, \Sigma_{P}\right)$ is around zero, changing sign from about -0.4 to 0.4 . The weak correlation between $P_{\text {Joule }}$ and $\Sigma_{P}$ inside the auroral tongue is not surprising, as the conductance gradients in this region are rather weak, as is evident in Fig. 9. Behind the second auroral tongue Joule heating and Pedersen conductance again anticorrelate, so that $\operatorname{corr}\left(P_{\text {Joule }}, \Sigma_{P}\right)$ reaches -0.5 around $02: 28 \mathrm{UT}$, when the trailing edge is in the middle of the analysis area.

\subsection{Comparison with CUTLASS measurements}

As explained in Sect. 2.4, the Finnish CUTLASS radar measured the line-of-sight plasma velocity during the omegaband event. We can calculate the same component of the $\boldsymbol{E} \times \boldsymbol{B}$ drift velocity from the local KRM results. The comparison between CUTLASS measurements and local KRM results is shown in Fig. 15 for 5 time steps in the interval 02:06-02:22 UT. In this comparison we use the emap conductance estimate as input in the local KRM method. The emap data area is covered by beams 4-10 of the CUTLASS radar, so that beam 4 intersects the area in the South-West corner and beam 10 in the North-East corner. The local KRM data shown in Fig. 15 is chosen from such time steps that correspond to the integration time of CUTLASS beam 7 (in the center of the analysis area).

The time series at Fig. 15 begins at 02:06 UT, when the eastern half of the area is filled with auroras related to the first auroral tongue (cf. Fig. 5. During the next time steps the omega-band propagates eastward, so that at 02:10 UT the western half of the analysis area is covered by the dark region of the omega-band, at 02:14 UT the whole area is in the dark region, at 02:18 UT the western half is covered by the second bright tongue and finally at 02:22 UT the tongue covers the whole analysis area.

We see in Fig. 15 that there is a good qualitative agreement between the CUTLASS measurement and our local KRM results. The plasma flow is first away from the radar, especially at the eastern part of the analysis area, indicating an eastward component of the electric field. The flow direction changes towards the radar when the dark inter-tongue region passes through the area around 02:14 UT, and finally inside the bright tongue the flow velocity is small, indicating also a suppressed electric field. This development is also 

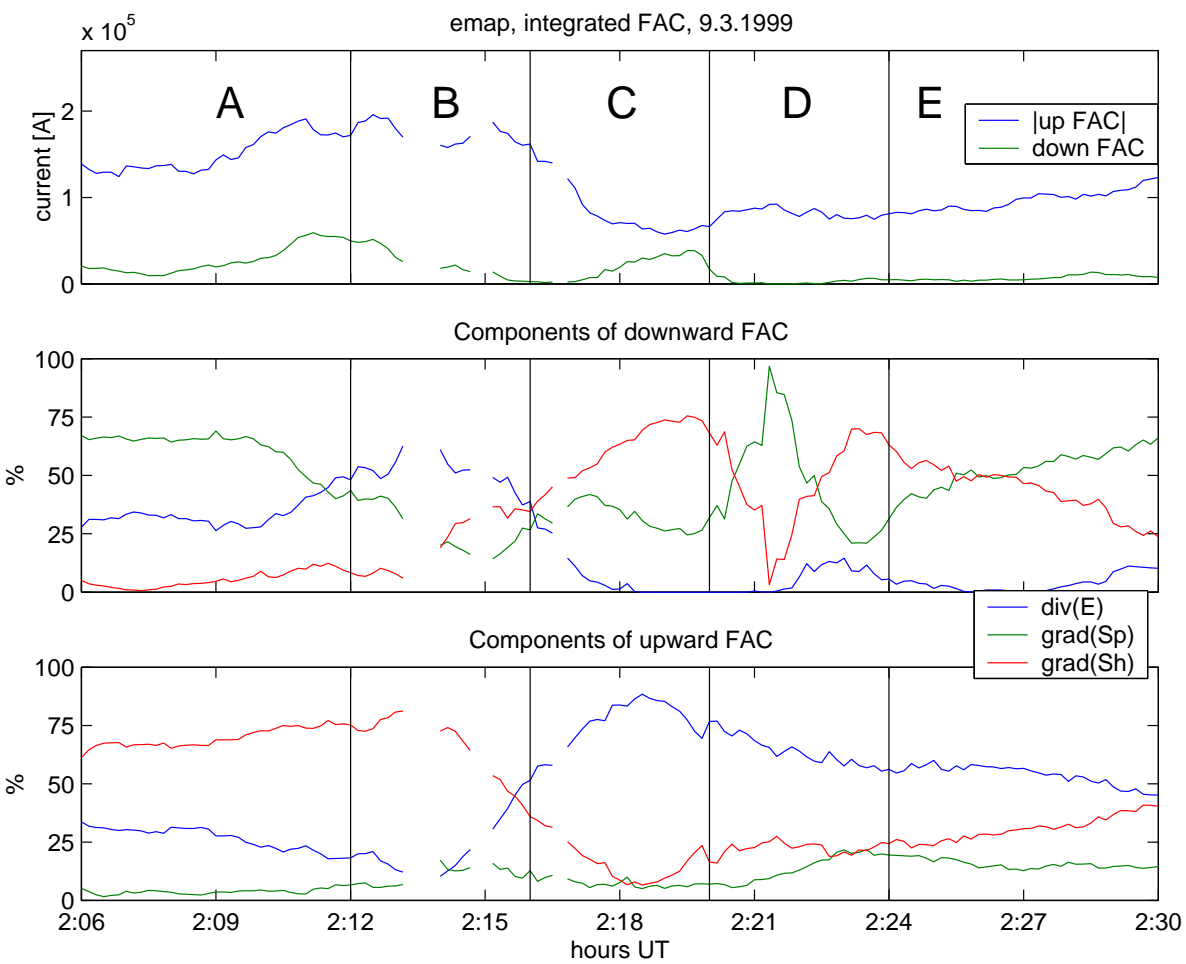

Fig. 12. Time series of the upward and downward FAC integrated over the emap data area, together with contributions from the different terms of $\nabla \cdot \boldsymbol{J}$. The upper panel gives the total upward and downward FAC. Middle panel shows the relative importance of the different components for the downward FAC, while bottom panel shows component of the upward FAC in similar manner. The vertical lines indicate the (approximate) intervals when different regions of the omega-band cross the analysis area, A: trailing edge of the previous tongue, B: dark region between the tongues, $\mathrm{C}$ : leading edge of the second auroral tongue, D: auroral tongue, E: trailing edge of the second tongue.

in accordance with the data shown in Figs. 7-9. There are some differences in the details between the local KRM results and CUTLASS measurements, e.g. in the North-East corner of the area at 02:18 UT and in the over-all flow velocity at 02:18 UT and 02:22 UT. However, these differences do not invalidate the general qualitative agreement.

\section{Discussion and conclusions}

We have studied the electrodynamics of an omega-band event with the local KRM method (Vanhamäki and Amm, 2007) that uses magnetometer, riometer and optical data as input and generates the electric field as output. Magnetometer data are used to estimate the ionospheric equivalent currents while optical and riometer data are used to estimate the ionospheric conductances. The electric field obtained from the local KRM method together with the conductance estimates gives the spatial distribution of ionospheric currents (including FAC) in the omega-band region. The omega-band sequence (9 March 1999, 01:45-02:30 UT) studied in this paper consists of two discernible auroral tongues, the first of which was mixed with some pseudo-breakup type features. We have used the second structure as a test case to evaluate the consistency of our local KRM results with earlier omegaband studies.

In this study we have combined ground-based magnetometer data with two separate conductance estimates that have complementary spatial resolution and extent. The satellite data (UVI estimate, based on the UVI instrument onboard POLAR) is used in a larger analysis area of $700 \times 600 \mathrm{~km}$ and has spatial resolution of $360 \mathrm{~km}$ in longitude and $40 \mathrm{~km}$ in latitude. The ground-based conductance data (emap estimate, based on ASC and riometer data) is available at a $220 \times 220 \mathrm{~km}$ area with about $10 \mathrm{~km}$ resolution. These conductance estimates have either been spatially interpolated (UVI) or averaged (emap) to the resolution of the equivalent current data $(\sim 50 \mathrm{~km})$. Even after averaging the higher spatial resolution of the emap conductance estimate over the equivalent current data may have caused some spurious small scale features to appear in the results. We have tried to identify those in the text. On the other hand, the lower spatial resolution of the UVI estimate may have resulted in loss of detail in the large scale results. Conductance gradients in the UVI estimate are rather weak $(\leq 0.05 \mathrm{~S} / \mathrm{km})$, which implies a dominating role for equivalent currents in defining the distributions of electric field and FAC. Overall, the 

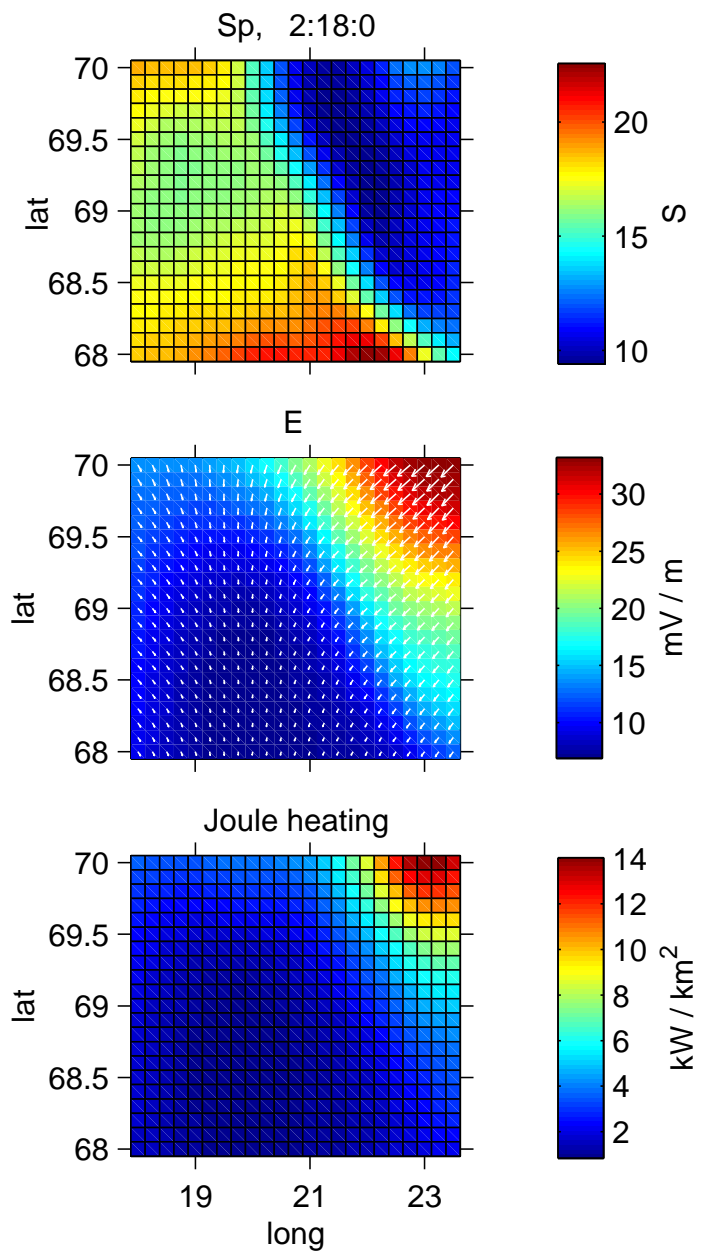

Fig. 13. From top to bottom: The Pedersen conductance, electric field (vector field and magnitude) and Joule heating $\boldsymbol{E} \cdot \boldsymbol{J}$. This snapshot corresponds to the situation illustrated in Fig. 8

UVI-based results of the omega-band current system are remarkably similar to the picture presented by Opgenoorth et al. (1983).

In our case the UVI-based conductance estimates are systematically smaller than those from combined ASC and riometer data (emap estimates). Therefore the electric field intensities in UVI-based results are roughly 1.5 times larger than from ground-based data $(\sim 50 \mathrm{mV} / \mathrm{m}$ versus $\sim 30 \mathrm{mV} / \mathrm{m}$ ). Previous studies do not help us in distinguishing which of these values is more correct since the STARE radar has measured electric field values consistent with both estimates near omega-bands (Opgenoorth et al., 1983; Amm et al., 2005). A test of the local KRM method with an artificial omega-band model reveals that the method tends to yield too small electric field values (Vanhamäki and Amm, 2007). Consequently we suspect that the electric field intensities deduced both from UVI and emap conductances are underestimates although the results from the latter method are surprisingly consistent with CUTLASS line-of-sight velocity measurements in the dark region of the omega-band. In this context it is important to remember that also different measurement techniques can yield different electric field estimates like in the case of Wild et al. (2000), where STARE measured significantly larger electric fields than CUTLASS did in the same region.

The electric field and current patterns based on emap results are more complicated than those from the UVI results. The equivalent current varies rather smoothly in scales $<200 \mathrm{~km}$, while the emap conductances show large gradients $(\leq 0.7 \mathrm{~S} / \mathrm{km})$ within the analysis area. Consequently, small scale structures appear also in our output data, especially in the FAC patterns. In the dark regions where the electric field is large even small changes in the conductance may cause large FACs. Such FAC structures should be interpreted with a grain of salt as they may be artefacts due to the different spatial resolution of the $\boldsymbol{J}_{\text {eq,ion }}$ and conductance data sets used as input. The edges of the auroral tongues are safer areas to discuss and there our results show features quite similar to those found by Wild et al. (2000). A sheet of downward (upward) FAC resides at the eastern (western) edge of the auroral tongue while FACs inside the tongue are generally smaller than at the edges. It is interesting to note that also the omega-band event studied by Wild et al. (2000) was accompanied with a simultaneous substorm intensification that occurred in the recovery phase of a previous substorm. Also the omega-band event studied in this paper was associated with pseudo-breakup type features.

The system of FAC sheets with alternating current directions observed in our event has a much smaller effect on the background electrojet than found in the omega-band event of Amm et al. (2005), where the westward electrojet current is almost completely interrupted at the auroral tongue. In our case the real horizontal currents follow quite closely the behaviour of the equivalent currents, i.e. they show an undulating pattern, instead of a "dashed line" type electrojet like in the case of Amm et al. (2005). The current density values in our FAC sheets are also much smaller than the values associated with the electrojet interruption. Also the spatial distribution of most intensive Joule heating regions is different in these two cases: while in the event of Amm et al. (2005) strongest heating takes place in the tongues of high conductance, in our event heating is stronger within the dark region than inside the auroral tongue. We notice, however, that the two tongues in our event have differences in this respect: the heating rates associated with the first auroral tongue are larger than those of the second tongue and of the dark region between the tongues. On the other hand, as the first tongue was combined with pseudo-breakup auroras its higher Joule heating rates when compared to the "pure" omega-band tongue are not surprising. The lesson to learn from our results is anyway that the spatial distribution of Joule heating in omega-bands cannot be deduced on the basis of optical data alone. 

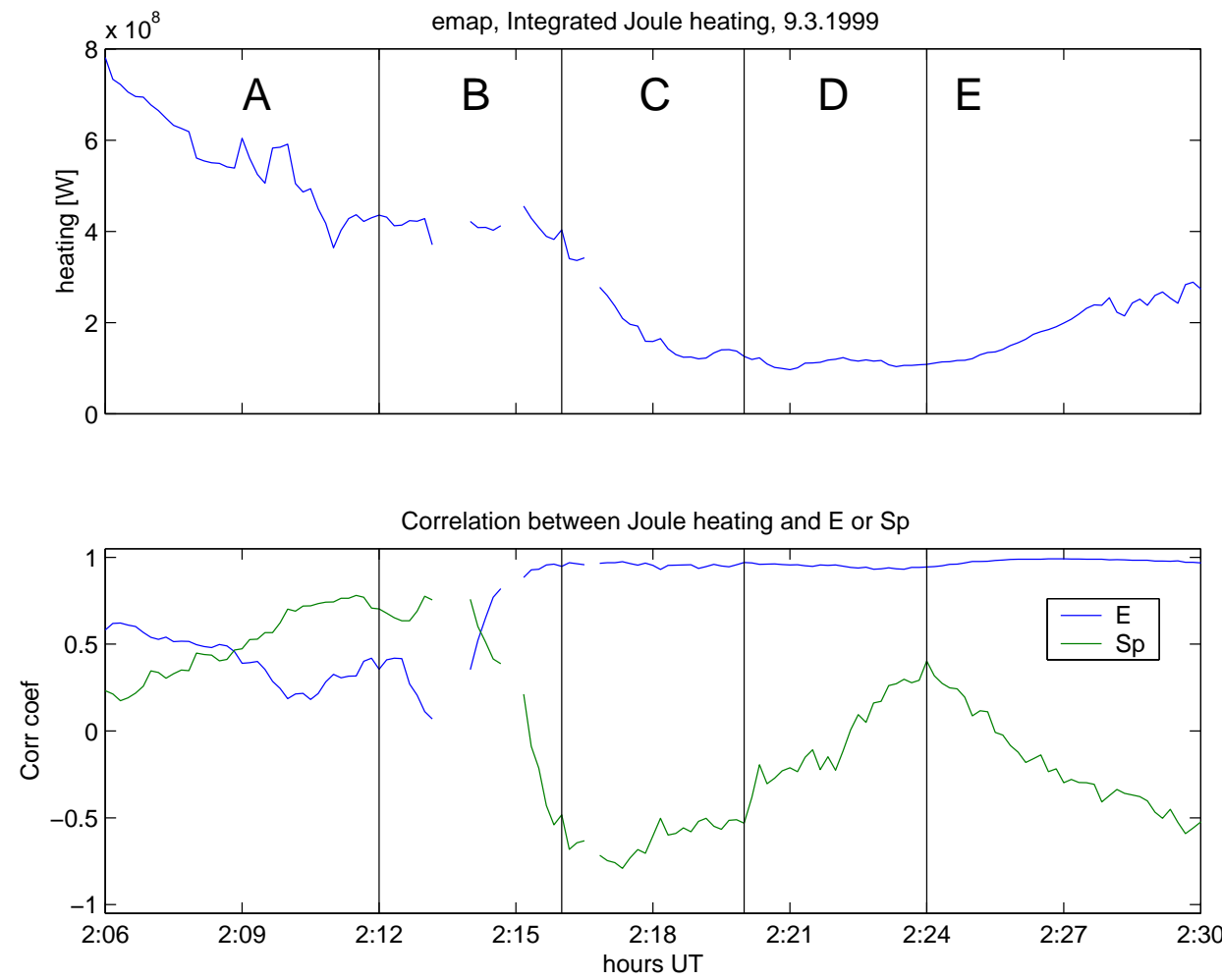

Fig. 14. Time series of the Joule heating (upper panel) and its correlation with the electric field and Pedersen conductance (lower panel). Correlation is calculated over the emap data area, separately for each time step. The different regions (A-E) of the omega-band are labelled as in Fig. 12.
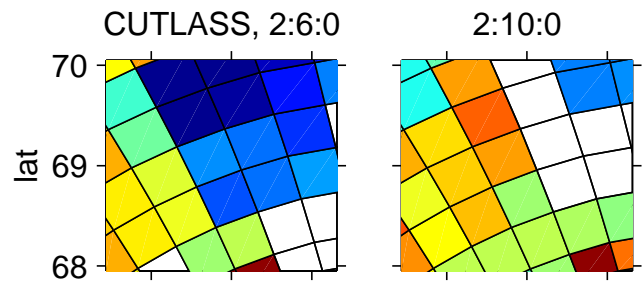

KRM, 2:6:50

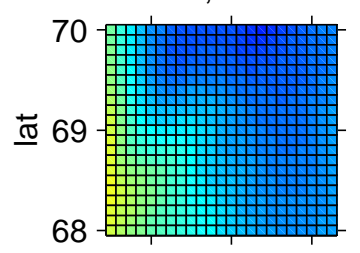

$\begin{array}{lll}19 & 21 \quad 23\end{array}$
2:10:50

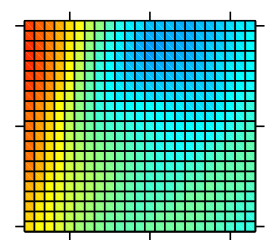

$19 \quad 21 \quad 23$

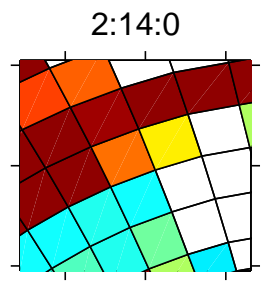

$2: 14: 40$

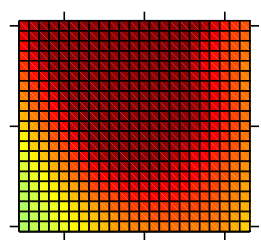

$19 \quad 21 \quad 23$

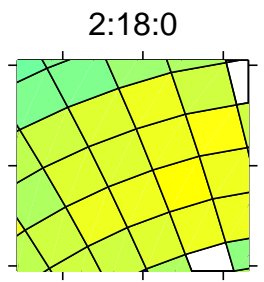

$2: 18: 50$

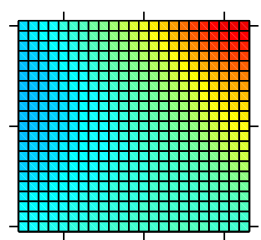

$19 \quad 21 \quad 23$

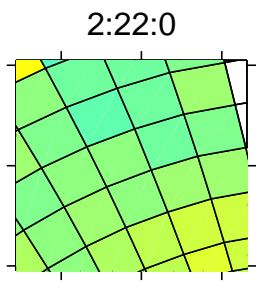

2:22:50

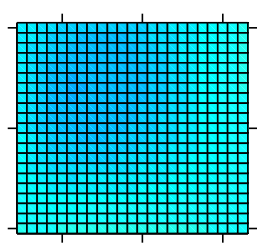

$\begin{array}{lll}19 & 21 & 23\end{array}$

Fig. 15. The line-of-sight plasma velocity measured by the Finnish CUTLASS radar and the same component of the $\boldsymbol{E} \times \boldsymbol{B}$ drift velocity calculated from the local KRM results. Comparison is shown for 5 time steps, so that the CUTLASS results are in the top row and corresponding local KRM results in the bottom row. Positive velocity is towards the radar, indicating that the ionospheric electric field has a westward component. 
The exercise conducted in this paper has demonstrated that the electrodynamics of omega-bands as deduced from opti$\mathrm{cal}$, riometer and magnetic data has the same main features as achieved with methods relying on radar observations. This gives some hope for more quantitative usage of all-sky camera data in the research of meso-scale electrodynamics associated with magnetosphere-ionosphere coupling phenomena. In addition to the spatial distribution of auroras also their intensities can be used when solving the ionospheric Ohm's law. Under certain conditions using auroral data instead of radar observations yields opportunities to see current and electric field patterns with improved spatial resolution (i.e. scale sizes below $50 \mathrm{~km}$ ). This opens new ways to investigate linkages between meso-scale and global phenomena in those regions where dense magnetometer and all-sky camera networks reside in the field-of-view of SuperDARN radars.

Acknowledgements. Work of $\mathrm{H}$. Vanhamäki was supported by Academy of Finland projects 115947 and 126552. The IMAGE magnetometer network is an international project coordinated by the Finnish Meteorological Institute (http://space.fmi.fi/image/). The IRIS and DASI data were supplied by Lancaster University. IRIS is funded by STFC.

Topical Editor M. Pinnock thanks I. McCrea for his help in evaluating this paper.

\section{References}

Aksnes, A., Stadsnes, J., Bjordal, J., Østgaard, N., Vondrak, R. R., Detrick, D. L., Rosenberg, T. J., Germany, G. A., and Chenette, D.: Instantaneous ionospheric global conductance maps during an isolated substorm, Ann. Geophys., 20, 1181-1191, 2002, http://www.ann-geophys.net/20/1181/2002/.

Amm, O.: Improved electrodynamic modeling of an omega band and analysis of its current system, J. Geophys. Res., 101, 26772683, 1996.

Amm, O. and Viljanen, A.: Ionospheric disturbance magnetic field continuation from the ground to the ionosphere using spherical elementary current systems, Earth, Planets and Space, 51, 431440, 1999.

Amm, O., Aksnes, A., Stadsnes, J., Østgaard, N., Vondrak, R. R., Germany, G. A., Lu, G., and Viljanen, A.: Mesoscale ionospheric electrodynamics of omega bands determined from ground-based electromagnetic and satellite optical observations, Ann. Geophys., 23, 325-342, 2005,

http://www.ann-geophys.net/23/325/2005/.

Baker, K. B. and Wing, S.: A New Magnetic Coordinate System for Conjugate Studies at High Latitudes, J. Geophys. Res., 94, 9139-9143, 1989.

Browne, S., Hargreaves, J. K., and Honary, B.: An imagining riometer for ionospheric studies, Electron. Commun. Eng., 7, 209217, 1995.

Gasda, S. and Richmond, A. D.: Longitudinal and interhemispheric variations of auroral ionospheric electrodynamics in a realistic geomagnetic field, J. Geophys. Res., 103, 4011-4021, 1998.

Germany, G. A., Parks, G. K., Brittnacher, M., Cumnock, J., Lummerzheim, D., Spann, J. F., Chen, L., Richards, P. G., and Rich,
F. J.: Remote determination of auroral energy characteristics during substorm activity, Geophys. Res. Lett., 24, 995-998, 1997.

Germany, G. A., Parks, G. K., Brittnacher, M. J., Spann, J. F., Cumnock, J., Lummerzheim, D., Rich, F., and Richards, P. G.: Energy Characterization of a Dynamic Auroral Event Using GGS UVI Images, in: Geospace Mass and Energy Flow, Geophysical Monograph 104, edited by: Horwitz, J. L., Gallagher, D. L., and Peterson, W., K., AGU, Washinton D.C., 143-147, 1998a.

Germany, G. A., Spann, J. F., Parks, G. K., Brittnacher, M. J., Elsen, R., Chen, L., Lummerzheim, D., and Rees, M. H.: Auroral Observations from the POLAR Ultraviolet Imager (UVI), in: Geospace Mass and Energy Flow, Geophysical Monograph 104, edited by: Horwitz, J. L., Gallagher, D. L., and Peterson, W. K., AGU, Washinton D.C., 149-159, 1998b.

Janhunen, P. and Huuskonen, A.: A numerical ionospheremagnetosphere coupling model with variable conductivities, J. Geophys. Res., 98, 9519-9530, 1993.

Kamide, Y., Richmond, A., and Matsushita, S.: Estimation of ionospheric electric fields, ionospheric currents, and field-aligned currents from ground magnetic records, J. Geophys. Res., 86, 801-813, 1981.

Kosch, M. J., Hagfors, T., and Nielsen, E.: A new digital all-sky imager experiment for optical auroral studies in conjunction with the Scandinavian twin auroral radar experiment, Rev. Sci. Inst., 69, 578-584, 1998.

Kosch, M. J., Honary, F., del Pozo, C. F., Marple, S. R., and Hagfors, T.: High-resolution maps of the characteristic energy of precipitating auroral particles, J. Geophys. Res., 106, 28925-28937, 2001.

Lester, M., Chapman, P. J., Cowley, S. W. H., et al.: Stereo CUTLASS - A new capability for the SuperDARN HF radars, Ann. Geophys., 22, 459-473, 2004, http://www.ann-geophys.net/22/459/2004/.

Murison, M., Richmond, A., Matsushita, S., and Baumjohann, W.: Estimation of ionospheric electric fields and currents from a regional magnetometer array, J. Geophys. Res., 90, 3525-3530, 1985.

Nielsen, E., Bruns, M., Pardowitz, I., Perplies, H., Bemmann, L., Janhunen, P., and Huuskonen, A.: STARE: Observations of a field-aligned line current, Geophys. Res. Lett., 26, 21-24, 1999.

Opgenoorth, H. J., Oksman, J., Kaila, K. U., Nielsen, E., Baumjohann, W.: Characteristics of eastward drifting omega bands in the morning sector aurora, J. Geophys. Res., 88, 9171-9185, 1983.

Opgenoorth, H. J., Persson, M. A. L., Pulkkinen, T. I., and Pellinen, R. J.: Recovery phase of magnetospheric substorms and its association with morning-sector aurora, J. Geophys. Res., 99, 41154129, 1994.

Pulkkinen, A., Amm, O., Viljanen, A., and BEAR Working Group: Ionospheric equivalent current distributions determined with the method of spherical elementary current systems, J. Geophys. Res., 108(A2), 1053, doi:10.1029/2001JA005085, 2003.

Richmond, A. D.: Ionospheric electrodynamics using magnetic apex coordinates, J. Geomag. Geoelectr., 47, 191-212, 1995.

Robinson, R. M., Vondrak, R. R., Miller, K., Dabbs, T., and Hardy, D.: On calculating ionospheric conductances from the flux and energy of precipitating electrons, J. Geophys. Res., 92, 25652569, 1987.

Senior, A., Kosch, M. J., and Honary, F.: Comparison of methods to determine auroral ionospheric conductances using ground-based 
optical and riometer data, Ann. Geophys., 26, 3831-3840, 2008, http://www.ann-geophys.net/26/3831/2008/.

Tanskanen, E., Viljanen, A., Pulkkinen, T., Pirjola, R., Häkkinen, L., Pulkkinen, A., and Amm, O.: At substorm onset $40 \%$ of AL comes from underground, J. Geophys. Res., 106(A7), 1311913134, 2001.

Untiedt, J. and Baumjohann, W.: Studies of polar current systems using the IMS Scandinavian magnetometer array, Space Sci. Rev., 63, 245-390, 1993.

Yamamoto, T., Inoue, S., and Meng C.-I.: Formation of auroral omega bands in the paired region 1 and region 2 field aligned current system, J. Geophys. Res., 102, 2531-2544, 1997.
Vanhamäki, H. and Amm, O.: A new method to estimate ionospheric electric fields and currents using data from a local ground magnetometer network, Ann. Geophys., 25, 1141-1156, 2007, http://www.ann-geophys.net/25/1141/2007/.

Vanhamäki, H., Amm, O., and Viljanen, A.: Role of inductive electric fields and currents in dynamical ionospheric situations, Ann. Geophys., 25, 437-455, 2007, http://www.ann-geophys.net/25/437/2007/.

Wild, J. A., Yeoman, T. K., Eglitis, P., and Opgenoorth, H. J.: Multiinstrument observations of the electric and magnetic field structure of omega bands, Ann. Geophys., 18, 99-110, 2000, http://www.ann-geophys.net/18/99/2000/. 